\title{
Satellitenbasierte Indexversicherungen zur Absicherung des witterungsbedingten Produktionsrisikos in der Land- und Forstwirtschaft
}

\author{
Dissertation \\ zur Erlangung des Doktorgrades (Dr. sc. agr.) \\ der Fakultät für Agrarwissenschaften \\ der Georg-August-Universität Göttingen

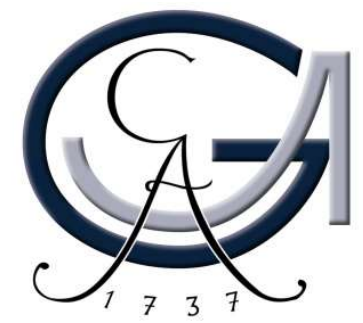

vorgelegt von

Wienand Kölle

geboren am 10.10.1991 in Hildesheim

Göttingen, im Juli 2021 
D7

1. Gutachter: Professor Dr. Oliver Mußhoff

2. Gutachter: Professor Dr. Matin Qaim

3. Gutachter: Professor Dr. Bernhard Möhring

Tag der mündlichen Prüfung: 27.09.2021 


\section{Danksagung}

Zu Beginn möchte ich mich bei meinen Eltern für die Unterstützung während meiner gesamten akademischen Ausbildung bedanken. Vor allem mein Vater hat mir durch die Übernahme der Hauptaufgaben auf dem landwirtschaftlichen Betrieb zuhause immer eine relativ große zeitliche Flexibilität ermöglicht.

Ganz besonderer Dank gilt Herrn Prof. Dr. Oliver Mußhoff für die gute Betreuung und insbesondere die zeitnahe Beantwortung und Diskussion von Fragen während der Promotion. Ferner möchte ich mich auch bei Prof. Dr. Matin Qaim und bei Prof. Dr. Bernhard Möhring für die Übernahme der Zweit- bzw. Drittprüferschaft bedanken. Aber auch Aoife Neville bin ich für die gute und schnelle Bearbeitung von Fragen und Problemen sehr dankbar.

Weiterhin möchte ich mich besonders bei Dr. Matthias Buchholz und Dr. Johannes Möllmann, die mich gut eingearbeitet haben und von denen ich gerade zu Beginn meiner Promotionszeit einiges lernen konnte, bedanken. Daneben möchte ich insbesondere Tilman Puls für seine Unterstützung bei Programmierproblemen und für den stets kritischen, thematischen Austausch danken.

Durch glückliche Umstände habe ich in meiner Promotionszeit das Büro mit Herrn Wilhelm Brandes (Prof. em. Dr. Wilhelm Brandes) teilen können. Zwischenzeitlich wurde unsere Zwei-Mann-Koalition um Alexander Günther ergänzt. Aber in der überwiegenden Zeit habe ich mich vormittags alleine mit Herrn Brandes über agrarspezifische, weltpolitische aber auch allgemeine Themen ausgetauscht. In dieser Zeit ist Herr Brandes zu einem großväterlichen Freund geworden, wodurch ich mich immer gerne an unsere Gespräche zurückerinnern werde.

Gerade im ersten Jahr meiner Promotion hat mir das gemeinsame Forschen mit Johannes Meyer und Johannes Möllmann sehr viel Spaß gemacht. Außerdem habe ich auch immer sehr gerne die Arbeitszeit mit meinem zeitweiligen Bürokollegen Alexander Günther verbracht. Aber auch die Unterhaltungen und gemeinsamen Pausen mit Carolin Winkel, Mira Knopp, Greta Langer, Felicia Leiffer, Christoph Wiedenroth, Louisa von Plettenberg, Henning Schaak, Luise Meißner, Vanessa Bonke, Annkathrin Possner, Selina Bruns, 
Cord-Friedrich von Hobe, Viktoria Graskemper, Aoife Neville und Andreas Heckman haben die Zeit während des Anfertigens der Doktorarbeit für mich bereichert. Besonders während der Coronazeit hat mir die Zusammenarbeit und der Austausch mit Sören Mohrmann und Eike Nordmeyer sehr viel Freude bereitet.

Darüber hinaus möchte ich mich bei Friedrich Stute und Dr. Constantin von Reitzenstein bedanken, die mir parallel zu meiner Promotion einen Einblick in das Management von landwirtschaftlichen Betrieben in Deutschland ermöglicht haben.

Vielen Dank! 


\section{Inhaltsverzeichnis}

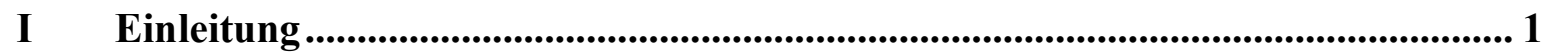

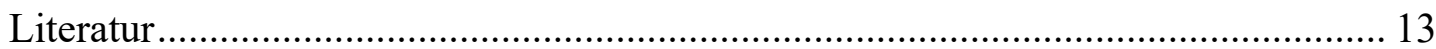

II Do remotely-sensed vegetation health indices explain credit risk in agricultural microfinance? ................................................................................................ 21

III Do high-resolution satellite indices at field level reduce basis risk of satellitebased weather index insurance? ..................................................................................... 22

IV Can satellite-based weather index insurance improve the hedging of yield risk of perennial non-irrigated olive trees in Spain?

V Can satellite-based weather index insurance hedge the mortality risk of pine stands?

VI Zusammenfassung und Schlussfolgerung ............................................................ 25

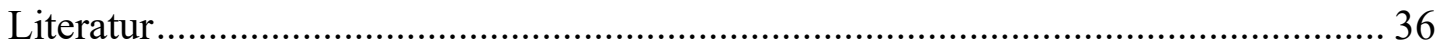

Publikationsliste............................................................................................................................... II

Erklärung über den geleisteten Eigenanteil an der Arbeit............................................. III

Eidesstattliche Erklärungen .................................................................................................... V 


\section{Einleitung}

„Sommer 2020 - staubtrockene Böden das dritte Jahr in Folge“

Diese Überschrift wählte der Deutsche Wetterdienst in einer Pressemitteilung für die Beschreibung des Wetters im Sommerhalbjahr 2020 (Deutscher Wetterdienst, 2021). Nach den bereits sehr trockenen Jahren 2018 und 2019 war auch das Frühjahr 2020 sehr niederschlagsarm, wodurch die Vegetationsperiode im Jahr 2020 ebenfalls durch Wasserknappheit und trockene Böden gekennzeichnet war. Solche zunehmend ungünstigen Wetterbedingungen wie Dürre stellen ein erhebliches Problem für die Land- und Forstwirtschaft dar. Bei geringen Niederschlägen und hohen Temperaturen sinken die Ernteerträge deutlich und somit werden auch unter dem voranschreitenden Klimawandel zukünftig Verluste in der Pflanzenproduktion prognostiziert (Chen und Gong, 2021; Matthew Gammans et al., 2017; Burke und Emerick, 2016). Ebenso stellen in der Forstwirtschaft die klimatischen Bedingungen und somit die Verfügbarkeit von Wasser die stärksten Beschränkungen für die Wachstumsrate und die Produktivität von Wäldern dar (Toledo et al., 2011; Orwig und Abrams, 1997). Trockenheitsbedingter Stress verändert die jährliche Wachstumsrate und die Baumsterblichkeit (Allen et al., 2010; Lo et al., 2010; van Mantgem et al., 2009), wodurch die wirtschaftliche Produktivität von Wäldern maßgeblich beeinflusst wird (Hanewinkel et al., 2013). Neben diesem Produktionsrisiko in Form von ungünstigen Wetterbedingungen tragen noch andere Faktoren dazu bei, dass vor allem die landwirtschaftliche Produktion in der heutigen Zeit eine risikoreiche ökonomische Aktivität ist. So führen institutionelle, politische und auch individuelle Risiken der Landwirte zu weiteren Risikoquellen in der landwirtschaftlichen Produktion (Bokusheva et al., 2016). Außerdem steigen die Marktrisiken im Zuge der Liberalisierung der Agrarmärkte und durch eine erhöhte Abhängigkeit von einer geringeren Anzahl an Lieferanten und Abnehmern in Folge eines Konzentrationsprozesses (Bahrs et al., 2004).

Diese veränderten Rahmenbedingungen führen zu einer zunehmenden Anspannung in der wirtschaftlichen Situation vieler landwirtschaftlicher Betriebe. Notwendige Anpassungen werden wie seit jeher nur durch Spezialisierung oder Wachstum bei vorausgesetzter Kosteneffizienz möglich sein. Entsprechend durchläuft die Landwirtschaft in der Europäischen Union (EU) seit Jahrzehnten einen spürbaren Strukturwandel (Neuenfeldt et al., 2019). Im 
Rahmen dieser strukturellen Entwicklungen ist die Zahl der landwirtschaftlichen Betriebe rückläufig, die Betriebsgröße wächst und es findet eine Spezialisierung in der Produktion und ein Entfernen von der gemischten Landwirtschaft statt (European Commission, 2013). Die aus dem Strukturwandel resultierenden Spezialisierungs- und Wachstumstendenzen führen häufig dazu, dass der Anteil an fremden Produktionsfaktoren (Arbeit, Boden, Kapital) in den landwirtschaftlichen Betrieben steigt (Schaper et al., 2011). In diesem Zusammenhang müssen Lohnarbeitskräfte, Pachtflächen und Fremdkapital regelmäßig entlohnt werden. Dadurch können längere Perioden niedriger Ernteerträge bzw. Erzeugerpreise weniger gut aufgefangen werden, wodurch das Existenzrisiko bei unvorhergesehenen Risiken steigt (Theuvsen, 2013). Somit sinkt aufgrund eines zunehmenden Einsatzes von fremden Produktionsfaktoren die Risikotragfähigkeit der landwirtschaftlichen Betriebe (Berg, 2005), wodurch wiederum die Notwendigkeit eines angemessenen Risikomanagements gerade vor dem Hintergrund jüngst steigender Wetterrisiken - aktueller denn je ist und vermutlich zukünftig noch steigen wird.

Speziell für die Wetterrisiken haben Landwirte zumeist einige Möglichkeiten, um die betriebliche Gefährdung durch ungünstige Wetterereignisse zu reduzieren. Neben der Nutzung von Bewässerungssystemen stellen auch die Bodenbearbeitung, der Anbau von dürreresistenten Sorten sowie Pflanzen mit kürzeren Produktionszyklen innerbetriebliche Anpassungsstrategien an das wetterbedingte Produktionsrisiko dar (Bokusheva et al., 2016). Allerdings könnten diese innerbetrieblichen Risikomanagementinstrumente zukünftig nicht mehr ausreichen, um den extremen Wetterereignissen der letzten Jahre zu begegnen. In solchen Situationen könnte der Rückgriff auf Versicherungslösungen interessant sein. In diesem Zusammenhang werden schon seit längerer Zeit sogenannte Wetterindexversicherungen im landwirtschaftlichen Bereich diskutiert, um wetterbedingte Ertragsschwankungen abzusichern (Leblois et al., 2014; Vedenov und Barnett, 2004; Turvey, 2001). Im Gegensatz zu schadensbasierten Versicherungen (wie z.B. die Hagelversicherung oder eine Mehrgefahrenversicherung gegen Hagel, Sturm, Starkregen aber auch Frost) können Wetterindexversicherungen dazu beitragen, die Probleme von Moral Hazard und der adversen Selektion zu reduzieren (Goodwin, 2001). Im Vergleich zu den üblichen schadensbasierten Versicherungen besitzen Wetterindexversicherungen u.a. den Vorteil, dass die Schadensfeststellung nicht durch einen Schadensschätzer erfolgt, wodurch Wetterindexversicherungen im Vergleich zu schadensbasierten Versicherungen geringere Transaktionskosten auf- 
weisen (Leblois et al., 2014). Vielmehr erfolgt die Schadensregulierung bei Wetterindexversicherungen anhand der Höhe eines objektiv messbaren Index. Somit kann die Höhe der Entschädigungszahlung direkt aus der Höhe des objektiv messbaren Index abgeleitet werden, indem eine Entschädigungszahlung erfolgt, sobald der Index einen gewissen Grenzwert (Strike-Level) unterschreitet bzw. übersteigt (Skees et al., 1997). Ursprünglich stammen Wetterindexversicherungen aus dem Energie- und Tourismussektor (Norton et al., 2012). Im landwirtschaftlichen Bereich sind Wetterindexversicherungen weniger stark verbreitet, da diese speziell in der landwirtschaftlichen Produktion mit einem Basisrisiko gepaart sind (Woodard und Garcia, 2008), welches in einem geringen Zusammenhang zwischen Index und Pflanzenertrag besteht (Turvey, 2001). Im Rahmen von Wetterindexversicherungen werden häufig objektiv messbare Wetterstationsdaten als zugrundeliegende Indizes verwendet. Mit steigender Entfernung zur nächstgelegenen Wetterstation erhöht sich allerdings das geografische Basisrisiko und somit die Wahrscheinlichkeit, dass sich das Wettergeschehen an der Wetterstation von dem am Ort der landwirtschaftlichen Produktion unterscheidet. Demzufolge hängt die Absicherungswirkung einer wetterstationsbasierten Wetterindexversicherung sehr stark von der Entfernung zur nächstgelegenen Wetterstation ab (Norton et al., 2012). Im Falle eines vorherrschenden Basisrisikos ist folglich auch das Risikoreduzierungspotential bzw. die Hedgingeffektivität einer Wetterindexversicherung geringer.

Immer häufiger werden Wetterindexversicherungen in letzter Vergangenheit als Dürreversicherungen seitens der Versicherungsanbieter angeboten. Die erhältlichen Dürreversicherungen von Unternehmen wie Hagelgilde (2021), Mecklenburgische (2021), München \& Magdeburger Agrar AG (2021), Vereinigte Hagel (2021), Versicherungskammer Bayern (2021) und Wetterheld (2021) basieren auf einem Niederschlagsindex, der an einer Wetterstation gemessen bzw. zwischen unterschiedlichen Wetterstationen auf einem Raster interpoliert wird. Dabei zahlen die Versicherungen, wenn entweder die Anzahl niederschlagsarmer Tage oder die Niederschlagsmenge ein vorher definiertes Strike-Level über- bzw. unterschreitet. Dabei kann das Dürrerisiko bei den meisten Versicherungsanbietern nur in Kombination zusammen mit Hagel versichert werden. Allerdings sind die angebotenen Dürreversicherungen aufgrund der hohen Versicherungsbeiträge für Landwirte häufig uninteressant. Ursächlich dafür ist, dass Trockenheit zu den sogenannten Kumulrisiken gehört, wodurch bei einem Schadenseintritt gleichzeitig mehrere Regionen betroffen sind und 
somit aufgrund der hohen Schadenssummen auch eine hohe Versicherungsprämie verlangt wird (GDV, 2020). Hinzu kommt, dass bei dem häufig verwendeten Niederschlagsindex das Problem besteht, dass Niederschlagsereignisse räumlich sehr heterogen auftreten (Norton et al., 2012), wodurch die Quantifizierung der Niederschlagsmenge über Wetterstationsdaten als herausfordernd und kritisch zu betrachten ist. Außerdem ist gerade in Regionen mit einer großen Distanz zwischen der Wetterstation und dem Ort der Produktion sowie insbesondere in Entwicklungsländern, die häufig ein geringes Netzwerk an Wetterstationen aufweisen, die Abhängigkeit der wetterstationsbasierten Indexversicherungen von der Entfernung zur nächsten Wetterstation problematisch (Meroni et al., 2013). Folglich hängt das Potential einer Wetterindexversicherung stark von der Verfügbarkeit eines kostengünstigen und zuverlässigen Index als Grundlage für die Bewertung eines Schadens ab.

Zusätzlich zu den an Wetterstationen gemessenen Daten können auch Indizes auf Grundlage von fernerkundeten Satellitenaufnahmen im Rahmen von Wetterindexversicherungen verwendet werden. Bei der Fernerkundung werden Bilder von Kameras erzeugt, die auf Satelliten oder Flugzeugen montiert sind, um großflächige Gebiete zu überwachen und Veränderungen und Variationen auf der Erdoberfläche zu erkennen. Hierdurch können Rückschlüsse auf die Vegetationsbedingungen gezogen werden, da Licht oder Strahlung je nach Zustand der Pflanze oder des Bodens unterschiedlich stark reflektiert oder absorbiert wird (USGS, 2020). Aufgrund ihrer Unabhängigkeit von Wetterstationen können Satellitenindizes eine interessante Option sein, um das geografische Basisrisiko von traditionellen Wetterindexversicherungen zu reduzieren. Darüber hinaus sind Satellitenindizes weltweit nahezu in Echtzeit verfügbar und teilweise kostenlos (Quiring und Ganesh, 2010). Dementsprechend werden satellitenbasierte Wetterindexversicherungen schon seit längerem in der Literatur diskutiert. Dabei wurde in der Vergangenheit häufig der Normalized Difference Vegetation Index (NDVI) als objektiv messbarer Index für das Design von Wetterindexversicherungen verwendet (Chantarat et al., 2013; Miranda und Farrin, 2012; Leblois et al., 2014; Makaudze und Miranda, 2010). Der NDVI quantifiziert die Menge an grüner Biomasse in jedem Pixel einer Satellitenaufnahme und zeigt somit die Dichte und Vitalität der pflanzlichen Vegetation an, wodurch Erkenntnisse über die photosynthetische Aktivität gezogen werden können. Allerdings ist der NDVI für die Verwendung als Index im Rahmen von Wetterindexversicherungen in seiner Aussagekraft nicht vollumfänglich, da Standorteffekte wie Boden, Topographie oder auch Nährstoffaufnahme nicht berücksichtigt 
werden (Turvey und Mclaurin, 2012). Diese standortspezifischen Anpassungen werden bei der Berechnung des Vegetation Condition Index (VCI), des Temperature Condition Index (TCI) und des Vegetation Health Index (VHI) berücksichtigt (Kogan et al., 2016). Während der VCI wie der NDVI über die Menge der grünen Biomasse den aktuellen Zustand der Vegetation beschreibt, spiegelt der TCI die Temperaturbedingungen wider. Auf Grundlage dessen setzt sich der VHI aus einer Kombination aus VCI und TCI zusammen. In Studien zur Absicherung des Ertragsrisikos von Winterweizen in Kasachstan (Bokusheva et al., 2016) und Deutschland (Möllmann et al., 2019) konnte die Eignung dieser Indizes für satellitenbasierte Wetterindexversicherungen bestätigt werden. Dabei konnten Möllmann et al. (2019) nachweisen, dass satellitenbasierte Indexversicherungskontrakte Versicherungskontrakten, die auf Grundlage von an Wetterstationen gemessenen Niederschlags- bzw. Temperaturindizes Entschädigungszahlungen leisten, bei der Absicherung des Risikos in der Winterweizenproduktion überlegen sind.

Dennoch beschränken sich die derzeitig am Markt angebotenen satellitenbasierten Wetterindexversicherungen auf Versicherungskontrakte für Grün- bzw. Weideland in Kanada, Spanien und Frankreich (Vroege et al., 2019). Demnach besteht hinsichtlich der Anwendung von satellitenbasierten Wetterindexversicherungen für Marktfruchtkulturen wie Winterweizen, aber auch für mehrjährige Kulturen der Land- und Forstwirtschaft weiterhin Forschungsbedarf. Vor diesem Hintergrund befasst sich diese Dissertation mit der Eignung von satellitenbasierten Vegetationsindizes für Wetterindexversicherungen. Dabei sollen die Untersuchungen über die Wirkung von satellitenbasierten Wetterindexversicherungen von einjährigen Kulturen (Reis, Weizen) bis hin zu langjährigen Kulturen der Landwirtschaft (Olivenbäume) sowie der Forstwirtschaft (Kiefern) reichen. Für das Risikoreduzierungspotential von Wetterindexversicherungskontrakten ist es allerdings von zentraler Bedeutung, das Basisrisiko möglichst gering zu halten. Ursächlich für das bestehende Basisrisiko von Wetterindexversicherungen in Form einer geringen Korrelation zwischen Wetterindex und Kultur- bzw. Betriebsertrag können folgende Aspekte sein:

a) die Entfernung zwischen dem Ort der Produktion und der Wetterstation (geografisches Basisrisiko (Woodard und Garcia, 2008)),

b) der Einfluss anderer Faktoren auf die Ertragsbildung als die Wettervariable (Basisrisiko der Produktion (Woodard und Garcia, 2008)), 
c) der jährlich variierende Zeitpunkt des Auftretens ertragsbeeinflussender Umstände (zeitliches Basisrisiko (Deng et al., 2007)),

d) der Einfluss der Methode zur Bestimmung der Abhängigkeit zwischen Wetterindex und Kulturertrag (Basisrisiko des Designs (Bokusheva, 2018))

e) und - speziell für Satellitenindizes - der Einfluss der Pixelgröße bzw. räumlichen Auflösung der Satellitenaufnahmen (Basisrisiko der räumlichen Auflösung (Möllmann et al., 2019)).

Somit ist es das Ziel dieser kumulativen Dissertation, das Basisrisiko von Wetterindexversicherungen durch die Verwendung von Satellitenindizes und ein entsprechendes Design der Versicherungskontrakte $\mathrm{zu}$ reduzieren und die Wirkung von satellitenbasierten Indexversicherungskontrakten für verschiedene Kulturen der Land- und Forstwirtschaft zu analysieren.

In diesem Zusammenhang beschäftigt sich der erste Beitrag in Kapitel II dieser Dissertation (,Do remotely-sensed vegetation health indices explain credit risk in agricultural microfinance?“", erschienen in World Development 127 (104771) (2020)) mit der allgemeinen Möglichkeit von satellitenbasierten Wetterindexversicherungen zur Absicherung des Kreditausfallrisikos eines Mikrofinanzinstitutes in Madagaskar. So besitzt die eingangs erwähnte Entlohnung der steigenden Anteile an Fremdfaktoren (Arbeit, Boden, Kapital) nicht nur in europäischen landwirtschaftlichen Betrieben eine hohe Bedeutung. Insbesondere in Entwicklungsländern ist der Zugang zu Fremdkapital eine wichtige Voraussetzung für die landwirtschaftliche Produktion. Doch gerade bei Auftreten von Starkwetterereignissen können die Kredite aufgrund fehlender Risikomanagementinstrumente häufig durch die Kleinbauern nicht mehr zurückgezahlt werden. Daher ist die Vergabe von Mikrokrediten an landwirtschaftliche Unternehmen für ein Mikrofinanzinstitut aufgrund einer höheren Kreditausfallwahrscheinlichkeit oftmals risikoreicher als an ein nicht-landwirtschaftliches Unternehmen (Giné und Yang, 2009; Miranda und Gonzalez-Vega, 2010). Beispielhaft für ein kommerzielles Mikrofinanzinstitut wird in dem ersten Beitrag die AccèsBanque Madagaskar (ABM, 2016) betrachtet. In dem Einzugsgebiet dieses Mikrofinanzinstitutes erwirtschaften die Kleinbauern ihr Einkommen vorrangig durch den Anbau und Verkauf von Reis (Minten et al., 2009). Dementsprechend hängt die Kreditrückzahlungsfähigkeit für die in Anspruch genommenen Kredite, die für die Betriebsmittel der Reisproduktion benötigt werden, stark von der Höhe der Reisernte ab. Eine Studie der Autoren von Negenborn et 
al. (2018) zeigt vor diesem Hintergrund einen Zusammenhang zwischen dem Kreditrisiko von landwirtschaftlichen Unternehmen und den an Wetterstationen gemessenen Niederschlags- bzw. Evapotranspirationsindizes. Doch gerade in Entwicklungsländern wie Madagaskar ist das Netzwerk an Wetterstationen häufig gering und auch die Verfügbarkeit von längerfristigen, kontinuierlichen Zeitreihen ist teilweise nicht sichergestellt (Meroni et al., 2013). Daher verwendet der erste Beitrag dieser Dissertation die Satellitenindizes VCI, TCI und VHI, um das Kreditausfallrisiko landwirtschaftlicher Kreditnehmer zu erklären. Neben ihrer Unabhängigkeit von Wetterstationen weisen der VCI, TCI und VHI eine hohe Korrelation zu den Reiserträgen auf (Rahman et al., 2009). Dementsprechend sollen in dem ersten Beitrag die zwei folgenden Forschungsfragen untersucht werden:

- Besitzen der VCI, TCI und VHI neben kredit- und unternehmerspezifischen Variablen eine Erklärungskraft für das relativ hohe Kreditausfallrisiko von reisanbauenden Kleinbauern der AccèsBanque Madagaskar?

- Besteht ein Zusammenhang zwischen der Höhe des Kreditausfallrisikos und der Höhe des VCI, TCI bzw. VHI?

Dabei wurden die Satellitenindizes VCI, TCI und VHI anhand von Advanced Very High Resolution Radiometer Satellitenaufnahmen mit einer räumlichen Auflösung von 4 x 4 km gebildet (NOAA/STAR, 1981). Daneben stammen die Kreditdaten mit der Kreditausfallwahrscheinlichkeit sowie den soziodemografischen Informationen aus einem Datensatz der AccèsBanque Madagaskar, der die Kreditdaten von vier verschiedenen Bankfilialen über einen Zeitraum von 2009 bis 2015 beinhaltet. Nachdem der jeweilige Satellitenindexberechnungszeitraum mit dem höchsten Spearman-Korrelationskoeffizienten zu dem Kreditausfallrisiko bestimmt wurde, wurden für die entsprechenden Reisfelder die aggregierten Satellitenindizes für die entsprechenden Zeiträume gebildet. Anschließend flossen die Satellitenindizes als unabhängige Variable neben den kredit- und unternehmensspezifischen Angaben in ein Sequential Logit Model zu Erklärung des Kreditausfallrisikos ein. Zusätzlich wurde jeweils eine Quantilsregression berechnet, um die Wirkung der Satellitenindexhöhe auf die Höhe des Kreditausfallrisikos zu untersuchen.

Allerdings ermöglicht die räumliche Auflösung der im ersten Beitrag verwendeten Satellitenaufnahmen von 4 x 4 km keine Bestimmung der Satellitenindizes gänzlich frei von störenden Landschaftselementen wie Bergen, Wäldern und Seen. Daher untersucht der zweite 
Beitrag in Kapitel III dieser Dissertation (,Do high-resolution satellite indices at field level reduce basis risk of satellite-based weather index insurance?", erschienen in Agricultural Finance Review, DOI: 10.1108/AFR-12-2020-0177) den Einfluss einer steigenden räumlichen Auflösung von Satellitenaufnahmen auf die Hedgingeffektivität von satellitenbasierten Wetterindexversicherungen zur Absicherung von Winterweizenerträgen. Eine Studie von Möllmann et al. (2019) definiert diesen Störfaktor als das Basisrisiko der räumlichen Auflösung, sodass mit steigender räumlicher Auflösung der Satellitenbilder der Anteil der bewirtschafteten Kulturfläche pro Pixel zunimmt. Allerdings wurde eine unterschiedliche räumliche Auflösung von Satellitendaten bisher nur im Zusammenhang mit Ertragsschätzungen von Getreide (Kumhálová und Matějková, 2017; Schut et al., 2009) und zur Landklassifizierung (Soares et al., 2008; Löw und Duveiller, 2014) untersucht. Vor diesem Hintergrund basiert die Datengrundlage des zweiten Beitrages auf MODerate-resolution Imaging Spectroradiometer (MODIS) und Landsat 5/8 Satellitenaufnahmen mit räumlichen Auflösungen von 250 x 250 m bzw. 30 x 30 m. Durch die hohe räumliche Auflösung der verwendeten Satellitenaufnahmen ist es nun erstmals möglich, den NDVI im Rahmen von Wetterindexversicherungen auf Schlagebene $\mathrm{zu}$ bestimmen. Vorangegangene Studien (Möllmann et al., 2019; Bokusheva et al., 2016) konnten aufgrund der geringeren räumlichen Auflösung der verwendeten Satellitenaufnahmen lediglich satellitenbasierte Wetterindexversicherungen auf Grundlage einer Satellitenindexberechnung auf Betriebsdurchschnittsebene mit derer auf Landkreisebene vergleichen. Die Ergebnisse deuten darauf hin, dass sich mit steigendem Aggregationslevel der Satellitendaten die Hedgingeffektivität von satellitenbasierten Wetterindexversicherungen erhöht. Dementsprechend untersucht der zweite Beitrag dieser Dissertation neben dem Einfluss der räumlichen Auflösung der Satellitenaufnahmen zusätzlich den Effekt des Aggregationslevels der Satelliten- sowie Winterweizenertragsdaten. Folglich sollen die folgenden Forschungsfragen beantwortet werden:

- Erhöht sich durch die Verwendung von höher auflösenden Satellitenaufnahmen die Hedgingeffektivität von satellitenbasierten Wetterindexversicherungen?

- Führt das Design von satellitenbasierten Wetterindexversicherungen auf Schlagebene anstatt auf Betriebsebene zu einer erhöhten Hedgingeffektivität? 
Die satellitenbasierten Wetterindexversicherungskontrakte auf Grundlage der MODIS und Landsat 5/8 Satellitenaufnahmen werden für drei Ackerbaubetriebe in Nordostdeutschland anhand aller bestellten Winterweizenfelder im Zeitraum von 2000 bis 2019 berechnet. Dabei wurde Nordostdeutschland als Untersuchungsgebiet gewählt, da insbesondere diese Region aufgrund sandiger Böden und relativ geringer Niederschläge einem erhöhten Wetterrisiko ausgesetzt ist. Der verwendete NDVI wurde jeweils auf Schlagebene und auf Betriebsdurchschnittsebene sowohl für die MODIS als auch Landsat 5/8 Satellitenaufnahmen für die verfügbaren Winterweizenfelder der entsprechenden Betriebe berechnet. Für die Berechnung der Hedgingeffektivitäten der Versicherungskontrakte wurden stochastische Simulationen, Quantilsregressionen (Conradt et al., 2015) sowie die Semivarianz als Risikomaß verwendet.

Im Gegensatz zu einjährigen Kulturen wie Reis oder Winterweizen kann bei mehrjährigen Kulturen die Produktion nicht so leicht an sich verändernde Rahmenbedingungen in Folge des Klimawandels angepasst werden (Gunathilaka et al., 2018b). Insbesondere die im Vorfeld benötigten hohen Kapitalkosten während des Anbaus, der häufig nicht bewässerte Anbau und die lange Lebensdauer von Plantagenkulturen erschweren die Anpassung an den Klimawandel (Gunathilaka et al., 2018a). Vor diesem Hintergrund betrachtet der dritte Beitrag in Kapitel IV dieser Dissertation (,,Can satellite-based weather index insurance improve the hedging of yield risk of perennial non-irrigated olive trees in Spain?", erschienen in Australian Journal of Agricultural and Resource Economics 65 (1): 66-93 (2020)) die Ertragsvariabilität von Olivenbäumen im südspanischen Andalusien. Obwohl Olivenbäume an geringe Niederschläge angepasst sind, haben sich die Schwankungen bei den Olivenölerträgen in den letzten 20 Jahren stark erhöht (Quiroga und Iglesias, 2009). Daher wird verstärkt die Bewässerung als Risikomanagementinstrument in Andalusien eingesetzt, um die Schwankungen der Olivenölerträge zu reduzieren. Allerdings führt der steigende Bedarf an Wasser für die Bewässerung zunehmend dazu, dass Wasser zu einer knappen Ressource wird (Martínez und Almonacid, 2017). In einer Studie beobachteten Salazar et al. (2019), dass die Risikomanagementinstrumente Bewässerung und Versicherung als Substitute verwendet werden können, wodurch ein negativer Zusammenhang zwischen der Nutzung einer Bewässerung und der Teilnahme in einem Versicherungsprogramm besteht. Folglich untersucht der dritte Beitrag dieser Dissertation die Möglichkeit von satellitenbasierten Indexversicherungskontrakten als Alternative zur Bewässerung, um 
das Olivenölertragsrisiko von nicht-bewässerten Olivenbäumen abzusichern. Neben den Satellitenindizes VCI, TCI und VHI werden ein wetterstationsbasierter Niederschlags- und ein Temperaturindex hinsichtlich ihrer Eignung untersucht, um unter anderem Rückschlüsse auf das geografische Basisrisiko ziehen zu können. Dazu sollen in dem dritten Beitrag die zwei folgenden Fragestellungen beantwortet werden:

- In welchen Vegetationsphasen besteht der größte Zusammenhang zwischen den satellitenbasierten bzw. meteorologischen Indizes und den Olivenölerträgen in Andalusien?

- Weisen satellitenbasierte Indexversicherungskontrakte eine höhere Hedgingeffektivität als wetterstationsbasierte Indexversicherungskontrakte zur Absicherung des Olivenölertrages von nicht-bewässerten Olivenbäumen in Andalusien auf?

Um die Fragestellungen zu beantworten, wurden der VCI, TCI und VHI auf Grundlage von MODIS Satellitenaufnahmen mit einer räumlichen Auflösung von 250 x 250 m verwendet. Daneben wurden die Ertragsdaten vom spanischen Ministerium für Landwirtschaft, Fischerei und Ernährung (Ministerio de Agricultura, Pesca y Alimentacíon) zur Verfügung gestellt, wobei der Datensatz die durchschnittlichen Olivenölerträge aller bewässerten und nicht-bewässerten Olivenbäume der Provinzen Cordoba, Granada, Jaen, Malaga und Sevilla enthält. Folglich beziehen sich die designten Indexversicherungskontrakte auf die jeweilige Provinzebene, da keine Olivenölerträge auf Betriebsebene für die Untersuchungen vorlagen. Allerdings konnten die einzelnen Olivenfelder in den jeweiligen Provinzen relativ genau identifiziert werden, wodurch die durchschnittlichen Satellitenindizes aller Olivenfelder einer Provinz berechnet werden konnten. Die meteorologischen Temperatur- und Niederschlagsdaten, die von der spanischen Meteorologischen Agentur (AEMET) und vom Institut für Forschung und Bildung für Landwirtschaft und Fischerei (IFAPA) der Junta de Andalucía stammen, wurden ebenfalls als Durchschnittswerte aus den Daten von allen verfügbaren Wetterstationen einer Provinz berechnet. Vor dem Hintergrund, dass Extremwetterbedingungen einen starken Einfluss auf den Olivenertrag haben (Oteros et al., 2013; Ozdemir, 2016), werden die Wetterindexversicherungskontrakte zur Absicherung gegen extreme Wetterbedingungen entworfen und designt. $\mathrm{Zu}$ diesem Zweck wird der Copula-Ansatz für eine angemessene und robuste Schätzung der Ertragsabhängigkeit von extremen Wetterbedingungen verwendet. Dieser verspricht, extreme Er- 
tragsverluste besser modellieren zu können, da Copulas im Vergleich zu der üblicherweise verwendeten Regressionsmethode, die eine lineare Beziehung zwischen Index und Ernteertrag annimmt, stochastische Abhängigkeiten deutlich flexibler darstellen können (Embrechts et al., 2010). Neben der Verwendung des Copula-Ansatzes, um das Basisrisikos des Designs zu reduzieren, wurden für die Bestimmung der Hedgingeffektivitäten der Versicherungskontrakte stochastische Simulationen sowie die Semivarianz als Risikomaß verwendet.

Auf Grundlage des dritten Beitrages überträgt der vierte Beitrag in Kapitel V dieser Dissertation (,,Can satellite-based weather index insurance hedge the mortality risk of pine stands? “, erschienen in Journal of Forest Economics Vol. 36: No. 4, pp 315-350) satellitenbasierte Wetterindexversicherungen von langjährigen Olivenbäumen auf langjährige Waldbestände. Durch steigende Temperaturen nimmt trockenheitsbedingter Stress in Wäldern zu (Williams et al., 2013; Xu et al., 2019), wodurch sich die jährlichen Wachstumsraten, die Baumsterblichkeit (van Mantgem et al., 2009; Allen et al., 2010; Lo et al., 2010) und schließlich die wirtschaftliche Produktivität von Wäldern ändern (Hanewinkel et al., 2013). Forstversicherungen als Risikomanagementinstrumente sind in der Literatur nur selten untersucht worden. In erster Linie wurden Versicherungen gegen Feuer (Pinheiro und Ribeiro, 2013; Barreal et al., 2014), Stürme (Brunette und Couture, 2008) oder gegen beide Elemente (Sauter et al., 2016) betrachtet. Obwohl in den letzten 70 Jahren die Schäden durch Dürre, Feuer, Sturm sowie Insekten und Krankheiten zugenommen haben, ist nur ein kleiner Teil des weltweiten Privatwaldes versichert. Vor allem die hohen Versicherungsprämien sind für Waldbesitzer nicht attraktiv (Brunette et al., 2015; Zhang und Stenger, 2014). Insbesondere die im Forstbereich höheren Transaktionskosten im Vergleich zu landwirtschaftlichen Gütern sind ursächlich für die höheren Prämien. Diese werden durch die Bewertung von stehendem Holz, die Messung von jährlichen Wachstumsraten und auch die notwendige Beobachtung von Marktbedingungen begründet (Zhang und Stenger, 2014). Hinzu kommt, dass öffentliche Entschädigungszahlungen bei größeren Schadensereignissen die Zahlungsbereitschaft der Waldbesitzer für Versicherungen zusätzlich reduzieren (Sauter et al., 2016). Daher könnten satellitenbasierte Indexversicherungen in der Forstwirtschaft dazu beitragen, die Versicherungsprämien zu senken, da keine Schadensregulierung notwendig ist und die teilweise kostenlosen Satellitenindizes auch für große Waldflächen gebildet werden können. Vor diesem Hintergrund betrachtet der vierte Bei- 
trag dieser Dissertation satellitenbasierte Indexversicherungen vergleichend zu wetterstationsbasierten Indexversicherungen im Forstbereich, um die Vitalität und das damit verbundene Mortalitätsrisiko von Waldbeständen abzusichern. Dazu dient in dieser Studie der jährliche Baumzuwachs als Indikator für das Mortalitätsrisiko. Der jährliche Baumzuwachs wird mit der Methode der Dendrochronologie gewonnen, die es ermöglicht, das jährliche Baumwachstum durch die Breite der Baumringe sehr genau zu messen (Spiecker, 2002; Fritts, 2001). Dabei können geringere Wachstumsraten mit einer geringeren Vitalität und einem erhöhten Mortalitätsrisiko in Verbindung gesetzt werden (Gillner et al., 2013). Die Daten der jährlichen Wachstumsraten der Bäume wurden vom Institut für Waldökosysteme des Thünen-Institutes (Bundesforschungsinstitut für ländliche Räume, Wald und Fischerei) zur Verfügung gestellt. Die sich verändernden Bedingungen im Wald werden im Rahmen des intensiven Waldumweltmonitorings (Level II) ermittelt. Im Zuge dieser Erhebungen werden die Veränderungen der Baumdurchmesser gemessen und damit das Waldwachstum ermittelt (Thünen Institute, 2020). In dem vierten Beitrag dieser Dissertation wurde die Absicherungsmöglichkeit für das Mortalitätsrisiko von zwölf Kiefernbeständen in Nordostdeutschland durch sowohl wetterstations- als auch satellitenbasierte Wetterindexversicherungen untersucht, um die folgenden Forschungsfragen beantworten zu können:

- In welchen jährlichen Vegetationsphasen der Kiefernbestände besteht der höchste Zusammenhang zwischen der jährlichen Baumvitalität und den meteorologischen bzw. satellitenbasierten Indizes?

- Weisen satellitenbasierte Indexversicherungskontrakte eine höhere Hedgingeffektivität als wetterstationsbasierte Indexversicherungskontrakte zur Absicherung des jährlichen Mortalitätsrisikos von Kiefernbeständen auf?

Während die verwendeten meteorologischen Indizes (Niederschlag, Temperatur sowie ein kombinierter Niederschlags-Temperatur-Index (PTI)) an den jeweilig nächstgelegenen Wetterstationen des Deutschen Wetterdienstes für die einzelnen Kiefernbestände gemessen wurden, wurden die verwendeten Satellitenindizes VCI, TCI und VHI auf der Grundlage von MODIS Satellitenaufnahmen mit einer räumlichen Auflösung von 250 x $250 \mathrm{~m}$ berechnet. Für die Berechnung der Hedgingeffektivitäten der Versicherungskontrakte wurden stochastische Simulationen, Quantilsregressionen (Conradt et al., 2015) sowie die Semivarianz und die Semistandardabweichung als Risikomaße verwendet. 


\section{Literatur}

ABM (2016): Annual Report. AccèsBanque Madagascar. Online verfügbar unter https://www.accesbanque.mg/wp-content/uploads/2018/01/171123-RA-AccèsBanque-2016_Correct.pdf, zuletzt geprüft am 06.05.2021.

Allen, C. D.; Macalady, A. K.; Chenchouni, H.; Bachelet, D.; McDowell, N.; Vennetier, M. et al. (2010): A global overview of drought and heat-induced tree mortality reveals emerging climate change risks for forests. In: Forest Ecology and Management 259 (4), S. 660-684. DOI: 10.1016/j.foreco.2009.09.001.

Bahrs, E.; Fuhrmann, R.; Muziol, O. (2004): Die künftige Finanzierung landwirtschaftlicher Betriebe. Finanzierungsformen und Anpassungsstrategien zur Optimierung der Finanzierung. In: Schriftenreihe der Landwirtschaftlichen Rentenbank, S. 7-49. Online verfügbar unter https://www.rentenbank.de/export/sites/rentenbank /dokumente/Rentenbank_Schriftenreihe_Band19_.pdf, zuletzt geprüft am 04.05.2021.

Barreal, J.; Loureiro, M. L.; Picos, J. (2014): On insurance as a tool for securing forest restoration after wildfires. In: Forest Policy and Economics 42 (2), S. 15-23. DOI: 10.1016/j.forpol.2014.02.001.

Berg, E. (2005): Integriertes Risikomanagement: Notwendigkeit und Konzepte für die Landwirtschaft. In: J. Deiter (Hg.): Agrarökonomie im Wandel. Bonn, S. 53-67.

Bokusheva, R.; Kogan, F.; Vitkovskaya, I.; Conradt, S.; Batyrbayeva, M. (2016): Satellitebased vegetation health indices as a criteria for insuring against drought-related yield losses. In: Agricultural and Forest Meteorology 220 (10), S. 200-206. DOI: 10.1016/j.agrformet.2015.12.066.

Bokusheva, R. (2018): Using copulas for rating weather index insurance contracts. In: Journal of Applied Statistics 45 (13), S. 2328-2356. DOI: 10.1080/02664763.2017.1420146.

Brunette, M.; Holecy, J.; Sedliak, M.; Tucek, J.; Hanewinkel, M. (2015): An actuarial model of forest insurance against multiple natural hazards in fir (Abies Alba Mill.) stands in Slovakia. In: Forest Policy and Economics 55 (2), S. 46-57. DOI: 10.1016/j.forpol.2015.03.001. 
Brunette, M.; Couture, S. (2008): Public compensation for windstorm damage reduces incentives for risk management investments. In: Forest Policy and Economics 10 (78), S. 491-499. DOI: 10.1016/j.forpol.2008.05.001.

Burke, M.; Emerick, K. (2016): Adaptation to Climate Change: Evidence from US Agriculture. In: American Economic Journal: Economic Policy 8 (3), S. 106-140. DOI: $10.1257 /$ pol.20130025.

Chantarat, S.; Mude, A. G.; Barrett, C. B.; Carter, M. R. (2013): Designing Index-Based Livestock Insurance for Managing Asset Risk in Northern Kenya. In: Journal of Risk and Insurance 80 (1), S. 205-237. DOI: 10.1111/j.1539-6975.2012.01463.x.

Chen, S.; Gong, B. (2021): Response and adaptation of agriculture to climate change: Evidence from China. In: Journal of Development Economics 148, S. 102557. DOI: 10.1016/j.jdeveco.2020.102557.

Conradt, S.; Finger, R.; Bokusheva, R. (2015): Tailored to the extremes: Quantile regression for index-based insurance contract design. In: Agricultural Economics 46 (4), S. 537-547. DOI: 10.1111/agec.12180.

Deng, X.; Barnett, B. J.; Vedenov, D. V.; West, J. W. (2007): Hedging dairy production losses using weather-based index insurance. In: Agricultural Economics 36 (2), S. 271-280. DOI: 10.1111/j.1574-0862.2007.00204.x.

Deutscher Wetterdienst (2021): Sommer 2020 - staubtrockene Böden das dritte Jahr in Folge. Online verfügbar unter https://www.dwd.de/DE/presse/ pressemitteilungen/DE/2020/20201014_agrarwetter_sommer2020_news.html, zuletzt geprüft am 27.01.2021.

Embrechts, P.; McNeil, A. J.; Straumann, D. (2010): Correlation and Dependence in Risk Management: Properties and Pitfalls. In: M. A. H. Dempster (Hg.): Risk Management: Cambridge University Press, S. 176-223.

European Commission (2013): Structure and dynamics of EU farms: changes, trends and policy relevance. EU Agricultural Economics Brief No. 9, Directorate-General for Agriculture and Rural Development, European Commission, 2013.

Fritts, H. C. (2001): Tree rings and climate. Reprint of second printing 1976. Caldwell, N.J: Blackburn Press.

GDV (2020): Ernteeinbußen wegen Dürre: Versicherer fordern mehr staatliche Unterstützung für Landwirte. Landwirtschaftliche Mehrgefahrenversicherung. Online ver- 
fügbar unter https://www.gdv.de/de/medien/aktuell/ernteeinbussen-wegen-duerre-versicherer-fordern-mehr-staatliche-unterstuetzung-fuer-landwirte-61362, zuletzt geprüft am 14.06.2020.

Gillner, S.; Rüger, N.; Roloff, A.; Berger, U. (2013): Low relative growth rates predict future mortality of common beech (Fagus sylvatica L.). In: Forest Ecology and Management 302, S. 372-378. DOI: 10.1016/j.foreco.2013.03.032.

Giné, X.; Yang, D. (2009): Insurance, credit, and technology adoption: Field experimental evidence from Malawi. In: Journal of Development Economics 89 (1), S. 1-11. DOI: 10.1016/j.jdeveco.2008.09.007.

Goodwin, B. K. (2001): Problems with Market Insurance in Agriculture. In: American Journal of Agricultural Economics 83 (3), S. 643-649.

Gunathilaka, R. P. D.; Smart, James C. R.; Fleming, C. M. (2018a): Adaptation to climate change in perennial cropping systems: Options, barriers and policy implications. In: Environmental Science \& Policy 82, S. 108-116. DOI: 10.1016/j.envsci.2018.01.011.

Gunathilaka, R. P. D.; Smart, J. C. R.; Fleming, C. M.; Hasan, S. (2018b): The impact of climate change on labour demand in the plantation sector: the case of tea production in Sri Lanka. In: Aust J Agric Resour Econ 62 (3), S. 480-500. DOI: $10.1111 / 1467-8489.12262$.

Hagelgilde (2021): Hagelgilde Plus AQUA FLEX. Flexibler Schutz gegen Trockenheit und Nässe mit Hagelgilde Plus AQUA FLEX. Online verfügbar unter https://www.hagelgilde.de/hagelgilde-plus-aqua-flex/, zuletzt geprüft am 04.05.2021.

Hanewinkel, M.; Cullmann, D. A.; Schelhaas, M.-J.; Nabuurs, G.-J.; Zimmermann, N. E. (2013): Climate change may cause severe loss in the economic value of European forest land. In: Nature Clim Change 3 (3), S. 203-207. DOI: 10.1038/nclimate1687.

Kogan, F.; Guo, W.; Strashnaia, A.; Kleshenko, A.; Chub, O.; Virchenko, O. (2016): Modelling and prediction of crop losses from NOAA polar-orbiting operational satellites. In: Geomatics, Natural Hazards and Risk 7 (3), S. 886-900. DOI: 10.1080/19475705.2015.1009178. 
Kumhálová, J.; Matějková, Š. (2017): Yield variability prediction by remote sensing sensors with different spatial resolution. In: International Agrophysics 31 (2), S. 195 202. DOI: 10.1515/intag-2016-0046.

Leblois, A.; Quirion, P.; Alhassane, A.; Traoré, S. (2014): Weather Index Drought Insurance: An Ex Ante Evaluation for Millet Growers in Niger. In: Environ Resource Econ 57 (4), S. 527-551. DOI: 10.1007/s10640-013-9641-3.

Lo, Y.-H.; Blanco, J. A.; Seely, B.; Welham, C.; Kimmins, J. P. (2010): Relationships between climate and tree radial growth in interior British Columbia, Canada. In: Forest Ecology and Management 259 (5), S. 932-942. DOI: 10.1016/ j.foreco.2009.11.033.

Löw, F.; Duveiller, G. (2014): Defining the Spatial Resolution Requirements for Crop Identification Using Optical Remote Sensing. In: Remote Sensing 6 (9), S. 9034 9063. DOI: $10.3390 /$ rs6099034.

Makaudze, E. M.; Miranda, M. J. (2010): Catastrophic drought insurance based on the remotely sensed normalised difference vegetation index for smallholder farmers in Zimbabwe. In: Agrekon 49 (4), S. 418-432. DOI: 10.1080/0303 1853.2010.526690.

Martínez, J. D. S.; Almonacid, A. G. (2017): Productivism and Post-Productivism in the Olive Groves of Southern Spain. In: Quaestiones Geographicae 36 (2), S. 57-69. DOI: 10.1515/quageo-2017-0015.

Matthew Gammans; P. M.; Ariel O.-B. (2017): Negative impacts of climate change on cereal yields: statistical evidence from France. In: Environ. Res. Lett. 12 (5), S. 54007. DOI: 10.1088/1748-9326/aa6b0c.

Mecklenburgische (2021): Hagel-/Mehrgefahren-Versicherung+Dürre-Zusatz-Versiche rung. Online verfügbar unter https:/www.mecklenburgische.de/pdfs /produkte/ kundeninformationen/landwirtschaft/hagel-mehrgefahrenversicherung_ kundeninfo.pdf, zuletzt geprüft am 04.05.2021.

Meroni, M. J.; Kayitakire, F.; Brown, M. (2013): Remote sensing of vegetation: potential applications for index insurance. In: R. Gommes und F. Kayitakire (Hg.): The challenges of index-based insurance for food security in developing countries. Luxembourg, S. 238-245. 
Minten, B.; Randrianarison, L.; Swinnen, J. F. M. (2009): Global Retail Chains and Poor Farmers: Evidence from Madagascar. In: World Development 37 (11), S. 17281741. DOI: 10.1016/j.worlddev.2008.08.024.

Miranda, M. J.; Farrin, K. (2012): Index Insurance for Developing Countries. In: Applied Economic Perspectives and Policy 34 (3), S. 391-427. DOI: 10.1093/aepp/pps031.

Miranda, M. J.; Gonzalez-Vega, C. (2010): Systemic Risk, Index Insurance, and Optimal Management of Agricultural Loan Portfolios in Developing Countries. In: American Journal of Agricultural Economics 93 (2), S. 399-406. DOI: 10.1093/ ajae/aaq109.

Möllmann, J.; Buchholz, M.; Mußhoff, O. (2019): Comparing the Hedging Effectiveness of Weather Derivatives Based on Remotely Sensed Vegetation Health Indices and Meteorological Indices. In: Weather, Climate, and Society 11 (1), S. 33-48. DOI: 10.1175/WCAS-D-17-0127.1.

München \& Magdeburger Agrar AG (2021): Absicherung Sturm, Starkregen, Frost und Trockenheit. Trockenheitsversicherung. Online verfügbar unter https:// www.mmagrar.de/produkte/pflanzenversicherungen/absicherung-sturm-starkregenfrost.html, zuletzt geprüft am 04.05.2021.

von Negenborn, F.; Weber, R.; Musshoff, O. (2018): Explaining weather-related credit risk with evapotranspiration and precipitation indices. In: $A F R 78$ (2), S. 246-261. DOI: 10.1108/AFR-07-2017-0058.

Neuenfeldt, S.; Gocht, A.; Heckelei, T.; Ciaian, P. (2019): Explaining farm structural change in the European agriculture: a novel analytical framework. In: European Review of Agricultural Economics 46 (5), S. 713-768. DOI: 10.1093/erae/jby037.

NOAA/STAR (1981): 4 km AVHRR-VHP data in GEO-TIFF format. STAR - global vegetation health products. Online verfügbar unter https://www.star.nesdis.noaa.gov /smcd/emb/vci/VH/vh_ftp.php, zuletzt geprüft am 06.05.2021.

Norton, M. T.; Turvey, C.; Osgood, D. (2012): Quantifying spatial basis risk for weather index insurance. In: The Journal of Risk Finance 14 (1), S. 20-34.

Orwig, D. A.; Abrams, M. D. (1997): Variation in radial growth responses to drought among species, site, and canopy strata. In: Trees 11 (8), S. 474. DOI: $10.1007 / \mathrm{s} 004680050110$. 
Oteros, J.; García-Mozo, H.; Hervás, C.; Galán, C. (2013): Biometeorological and autoregressive indices for predicting olive pollen intensity. In: International journal of biometeorology 57 (2), S. 307-316. DOI: 10.1007/s00484-012-0555-5.

Ozdemir, Y. (2016): Effects of climate change on olive cultivation and table olive and olive oil quality. Scientific Papers. Series B, Horticulture, Volume LX (Online ISSN 2286-1580). Online verfügbar unter http://horticulturejournal.usa mv.ro/index.php/ scientific-papers/9-articles/412-effects-of-climate-change-on-olive -cultivation-andtable-olive-and-olive-oil-quality-412\#spucontentCitation11, zuletzt geprüft am 30.05.2020.

Pinheiro, A. C.; Ribeiro, N. A. (2013): Forest property insurance: an application to Portuguese woodlands. In: IJSSOC 5 (3), S. 284. DOI: 10.1504/IJSSOC.2013.054716.

Quiring, S. M.; Ganesh, S. (2010): Evaluating the utility of the Vegetation Condition Index (VCI) for monitoring meteorological drought in Texas. In: Agricultural and Forest Meteorology 150 (3), S. 330-339. DOI: 10.1016/j.agrformet.2009.11.015.

Quiroga, S.; Iglesias, A. (2009): A comparison of the climate risks of cereal, citrus, grapevine and olive production in Spain. In: Agricultural Systems 101 (1-2), S. 91-100. DOI: $10.1016 /$ j.agsy.2009.03.006.

Rahman, A.; Roytman, L.; Krakauer, N. Y.; Nizamuddin, M.; Goldberg, M. (2009): Use of vegetation health data for estimation of aus rice yield in bangladesh. In: Sensors (Basel, Switzerland) 9 (4), S. 2968-2975. DOI: 10.3390/s90402968.

Salazar, C.; Jaime, M.; Pinto, C.; Acuña, A. (2019): Interaction between crop insurance and technology adoption decisions: The case of wheat farmers in Chile. In: Aust $J$ Agric Resour Econ 4, S. 290. DOI: 10.1111/1467-8489.12307.

Sauter, P. A.; Möllmann, T. B.; Anastassiadis, F.; Mußhoff, O.; Möhring, B. (2016): To insure or not to insure? Analysis of foresters' willingness-to-pay for fire and storm insurance. In: Forest Policy and Economics 73 (2), S. 78-89. DOI: 10.1016/j.forpol.2016.08.005.

Schaper, C.; Deimel, M.; Theuvsen, L. (2011): Determinanten der Wettbewerbsfähigkeit „erweiterter Familienbetriebe“ - Ergebnisse einer Betriebsleiterbefragung. In: German Journal of Agricultural Economics 60 (1), S. 36-51. DOI: 10.22004/ag.econ.169832. 
Schut, A. G. T.; Stephens, D. J.; Stovold, R. G. H.; Adams, M.; Craig, R. L. (2009): Improved wheat yield and production forecasting with a moisture stress index, AVHRR and MODIS data. In: Crop Pasture Sci. 60 (1), S. 60. DOI: 10.1071/CP08182.

Skees, J. R.; Black, J. R.; Barnett, B. J. (1997): Designing and Rating an Area Yield Crop Insurance Contract. In: American Journal of Agricultural Economics 79 (2), S. 430-438. DOI: 10.2307/1244141.

Soares, D. M.; Galvão, L. S.; Formaggio, A. R. (2008): Crop area estimate from original and simulated spatial resolution data and landscape metrics. In: Sci. agric. (Piracicaba, Braz.) 65 (5), S. 459-467. DOI: 10.1590/S0103-90162008000500003.

Spiecker, H. (2002): Tree rings and forest management in Europe. In: Dendrochronologia 20 (1-2), S. 191-202. DOI: 10.1078/1125-7865-00016.

Theuvsen, L. (2013): Risks and Risk Management in Agriculture. In: Problemy Rolnictwa Światowego 13 [28] (4). Online verfügbar unter http://cejsh.icm.edu.pl/cejsh /element/bwmeta1.element.cejsh-from-agro-3b7b8932-c11c-41d3-af00-9c1 $\mathrm{d} 7 \mathrm{~b} 3 \mathrm{ba} 250$.

Thünen Institute (2020): Intensive Forest Environmental Monitoring (Level II). Forest growth. Online verfügbar unter https://blumwald.thuenen.de/level-ii/allgemeineinformationen/erhebungen/zuwachs-ertrag/, zuletzt geprüft am 17.02.2020.

Toledo, M.; Poorter, L.; Peña-Claros, M.; Alarcón, A.; Balcázar, J.; Leaño, C. et al. (2011): Climate is a stronger driver of tree and forest growth rates than soil and disturbance. In: Journal of Ecology 99 (1), S. 254-264. DOI: 10.1111/j.13652745.2010.01741.x.

Turvey, C. G.; Mclaurin, M. K. (2012): Applicability of the normalized difference vegetation index (NDVI) in index-based crop insurance design. In: Weather, Climate, and Society 4 (4), S. 271-284.

Turvey, C. G. (2001): Weather Derivatives for Specific Event Risks in Agriculture. In: Rev Agricultural Economics 23 (2), S. 333-351. DOI: 10.1111/1467-9353.00065.

USGS (2020): Mapping, Remote Sensing, and Geospatial Data. Online verfügbar unter https://www.usgs.gov/faqs/what-remote-sensing-and-what-it-used?qt-news_science _products=0\#qt-news_science_products, zuletzt geprüft am 17.11.2020. 
van Mantgem, P. J.; Stephenson, N. L.; Byrne, J. C.; Daniels, L. D.; Franklin, J. F.; Fulé, P. Z. et al. (2009): Widespread increase of tree mortality rates in the western United States. In: Science (New York, N.Y.) 323 (5913), S. 521-524. DOI: 10.1126/science. 1165000 .

Vedenov, D. V.; Barnett, B. J. (2004): Efficiency of weather derivatives as primary crop insurance instruments. In: Journal of Agricultural Resource Economics 29 (3), S. $387-403$.

Vereinigte Hagel (2021): FarmIndex Dürre N. Online verfügbar unter https:// www.vereinigte-hagel.net/wp-content/uploads/2020/09/Flyer_6-Seiter_Du\% CC\% 88rre_2020_ansicht.pdf, zuletzt geprüft am 04.05.2021.

Versicherungskammer Bayern (2021): ErnteSchutz Vario. Trockenheit. Online verfügbar unter https://www.vkb.de/content/firmen-landwirte/landwirte/ernteversicherungernteschutz/, zuletzt geprüft am 04.05.2021.

Vroege, W.; Dalhaus, T.; Finger, R. (2019): Index insurances for grasslands - A review for Europe and North-America. In: Agricultural Systems 168, S. 101-111. DOI: 10.1016/j.agsy.2018.10.009.

Wetterheld (2021): Gegen Dürre versichern. Online verfügbar unter https://wetterheld.com/home/de/drought/, zuletzt geprüft am 04.05.2021.

Williams, A. P.; Allen, C. D.; Macalady, A. K.; Griffin, D.; Woodhouse, C. A.; Meko, D. M. et al. (2013): Temperature as a potent driver of regional forest drought stress and tree mortality. In: Nature Clim Change 3 (3), S. 292-297. DOI: 10.1038/nclimate1693.

Woodard, J. D.; Garcia, P. (2008): Basis risk and weather hedging effectiveness. In: Agricultural Finance Review 68 (1), S. 99-117.

Xu, C.; McDowell, N. G.; Fisher, R. A.; Wei, L.; Sevanto, S.; Christoffersen, B. O. et al. (2019): Increasing impacts of extreme droughts on vegetation productivity under climate change. In: Nature Clim Change 9 (12), S. 948-953. DOI: 10.1038/s41558019-0630-6.

Zhang, D.; Stenger, A. (2014): Timber insurance: perspectives from a legal case and a preliminary review of practices throughout the world. In: New Zealand J For Sci 44 (Suppl 1), S9. DOI: 10.1186/1179-5395-44-S1-S9. 


\title{
II Do remotely-sensed vegetation health indices explain credit risk in agricultural microfinance?
}

\author{
Autoren: Johannes Möllmann, Matthias Buchholz, Wienand Kölle, Oliver Mußhoff \\ Erschienen in: World Development, Volume 127, March 2020, 104771,
} https://doi.org/10.1016/j.worlddev.2019.104771.

\begin{abstract}
Farmers' vulnerability to adverse weather events, which are likely to increase in frequency and magnitude due to climate change, is a major impediment to a sufficient credit supply. Smallholder farmers' access to credit is, among other factors, crucial for productivity and output growth. Index insurance could help lenders to compensate for lacking installment payments in years with severe weather conditions and, thus, is considered to accelerate agricultural lending. Using a unique borrower dataset provided by a Microfinance Institution (MFI) in Madagascar, we analyze whether remotely-sensed vegetation health indices can explain the credit risk of the MFI's agricultural loan portfolio. Therefore, we utilize sequential logit models and quantile regressions. More specifically, we consider the remotely-sensed Vegetation Condition Index, Temperature Condition Index and the Vegetation Health Index as independent variables at the individual branch and the aggregated bank level. These indices are available globally and can potentially enhance the effectiveness of index insurance by reducing basis risk (imperfect correlation between the index and the underlying exposure), a major drawback of index insurance. Moreover, we consider loan- and socio-demographic variables of the borrowers as additional independent variables. Our results show that the credit risk of the MFI is explained, to a large extent, by the vegetation health indices. Moreover, the results from quantile regressions show that the explanatory power of the vegetation health indices increases with increasing credit risk. Thus, utilizing remotely-sensed vegetation health indices for index insurance designs might be particularly valuable for MFIs to hedge the credit risk of their agricultural loan portfolio. Facing lower default rates, MFIs could reduce interest rates. Remotely-sensed index insurance could therefore enhance access to credit, contributing to sustainable development in the study region.
\end{abstract}

Keywords: Remotely-sensed data, Vegetation Health Indices, Credit risk, Microcredit, Index insurance. 


\title{
III Do high-resolution satellite indices at field level reduce basis risk of satellite-based weather index insurance?
}

\author{
Autoren: Wienand Kölle, Matthias Buchholz and Oliver Mußhoff \\ Erschienen in: Agricultural Finance Review,
} https://doi.org/10.1108/AFR-12-2020-0177.

\begin{abstract}
Purpose - Satellite-based weather index insurance has recently been considered in order to reduce the high basis risk of station-based weather index insurance. However, the use of satellite data with a relatively low spatial resolution has not yet made it possible to determine the satellite indices free of disturbing landscape elements such as mountains, forests and lakes.

Design/methodology/approach - In this context, the Normalized Difference Vegetation Index (NDVI) was used based on both Moderate Resolution Imaging Spectroradiometer (MODIS) $(250 \times 250 \mathrm{~m})$ and high-resolution Landsat 5/8 $(30 \times 30 \mathrm{~m})$ images to investigate the effect of a higher spatial resolution of satellite-based weather index contracts for hedging winter wheat yields. For three farms in north-east Germany, insurance contracts both at field and farm level were designed.

Findings - The results indicate that with an increasing spatial resolution of satellite data, the basis risk of satellite-based weather index insurance contracts can be reduced. However, the results also show that the design of NDVI-based insurance contracts at farm level also reduces the basis risk compared to field level. The study shows that higher-resolution satellite data is advantageous, whereas satellite indices at field level do not reduce the basis risk.

Originality - To the best of the author's knowledge, the effect of increasing spatial resolution of satellite images for satellite-based weather index insurance is investigated for the first time at the field level compared to the farm level.
\end{abstract}

Keywords - Weather index insurance, remote sensing, spatial resolution, data aggregation.

Article classification - Research paper 


\title{
IV Can satellite-based weather index insurance improve the hedging of yield risk of perennial non-irrigated olive trees in Spain?
}

\section{Autoren: Wienand Kölle, Andrea Martínez Salgueiro, Matthias Buchholz and Oliver Mußhoff}

Erschienen in: Australian Journal of Agricultural and Resource Economics, Volume 65, Issue 1, January 2021, Pages 66-93, https://doi.org/10.1111/14678489.12403.

\begin{abstract}
Olive oil yields fluctuate strongly due to their dependence on sufficient precipitation. An interesting option to hedge the yield risk in olive cultivation could be satellite-based weather index insurance. Therefore, we implement index insurance as a hedging alternative for non-irrigated olive groves using MODerate-resolution Imaging Spectroradiometer (MODIS) satellite data. For this purpose, we focus on the Spanish region of Andalusia, given its importance in olive production at the international level. We calculate three satellite indices: the Vegetation Condition Index (VCI), the Temperature Condition Index (TCI) and the Vegetation Health Index (VHI). Meteorological indices related to temperature and precipitation are used as benchmarks. Firstly, we estimate the periods that have the greatest influence on the critical vegetative phase of olives, which extends from March to September. Based on the indices, insurance contracts are designed using a copula approach, which is then employed to evaluate their hedging effectiveness. On average, the hedging effectiveness of VCI-, VHI- and TCI-based weather index insurance contracts amounts to 38 per cent, 38 per cent and 29 per cent, respectively. Moreover, VCI- and VHI-based weather index insurance contracts outperform traditional weather index insurance contracts based on precipitation (by 29 per cent) and temperature (by 16 per cent) indices.
\end{abstract}

Key words: copulas, olive trees, remotely sensed data, risk management, tail dependence, weather index insurance. 


\title{
V Can satellite-based weather index insurance hedge the mortality risk of pine stands?
}

\author{
Autoren: Wienand Kölle, Matthias Buchholz and Oliver Mußhoff \\ Erschienen in: Journal of Forest Economics, Vol. 36: No. 4, pp 315-350; \\ http://dx.doi.org/10.1561/112.00000533.
}

\begin{abstract}
Due to rising temperatures, forest stands are increasingly exposed to drought stress, which affects forest productivity through lower growth rates and higher mortality rates. Satellitebased index insurance could be an option for hedging the mortality risk of trees. Therefore, we calculated three remotely-sensed vegetation health indices from MODerate-resolution Imaging Spectroradiometer (MODIS) satellite images. As indices for index insurance we use the Vegetation Condition Index (VCI), the Temperature Condition Index (TCI) and the Vegetation Health Index (VHI). We generate temperature, precipitation and a combined index of the two as benchmark indices. For 12 pine stands in northeastern Germany, we calculate the hedging effectiveness for hypothetically designed index insurance contracts based on satellite and meteorological indices. Both station- and satellite-based insurance contracts have been shown to be effective in hedging standing timber mortality risk. The average hedging effectiveness ranges from $36 \%$ for TCI-based to $48 \%$ for VHI-based index insurance contracts.
\end{abstract}




\section{Zusammenfassung und Schlussfolgerung}

Länger anhaltende Extremwetterperioden haben die letzten Jahre geprägt. Neben den spürbaren zunehmenden Klimarisiken steigt auch das Marktrisiko aufgrund der Liberalisierung der Agrarmärkte. Diese veränderten Rahmenbedingungen stellen hohe Anforderungen an das Risikomanagement landwirtschaftlicher Betriebe, da gerade in Zeiten des Strukturwandels die Entlohnung eines zunehmenden Anteils an fremden Produktionsfaktoren (Arbeit, Boden, Kapital) die Risikotragfähigkeit der Betriebe reduziert. Vor diesem Hintergrund beschäftigt sich die vorliegende Dissertation mit satellitenbasierten Wetterindexversicherungen als Risikomanagementinstrument zur Absicherung des witterungsbedingten Produktionsrisikos in der Land- und Forstwirtschaft. Hierzu wird die Absicherungswirkung von satellitenbasierten Wetterindexversicherungen sowohl für einjährige landwirtschaftliche Kulturen (Reis, Weizen) als auch für langjährige Olivenbäume der Landwirtschaft sowie langjährige Kiefernbestände der Forstwirtschaft untersucht. Weiterhin werden in den einzelnen Beiträgen dieser Dissertation verschiedene Formen des bestehenden Basisrisikos von Wetterindexversicherungen betrachtet. In diesem Zusammenhang betrachtet der erste Beitrag satellitenbasierte Wetterindexversicherungen zur Absicherung des hohen Kreditausfallrisikos von Mikrokrediten für reisanbauende Kleinbauern in Madagaskar. Dagegen untersucht der zweite Beitrag die Wirkung von räumlich höher auflösenden Satellitenaufnahmen und den Effekt des Datenaggregationslevels im Rahmen von satellitenbasierten Wetterindexversicherungen zur Absicherung des Winterweizenertrages von landwirtschaftlichen Betrieben in Nordostdeutschland. Der dritte Beitrag beleuchtet satellitenbasierte Wetterindexversicherungskontrakte zur Absicherung des Olivenölertragsrisikos von nichtbewässerten Olivenbäumen in Andalusien als Alternative zur Bewässerung. Hingegen überträgt der vierte Beitrag satellitenbasierte Wetterindexversicherungen auf den Forstbereich und überprüft die Eignung solcher Versicherungskontrakte für die Absicherung des jährlichen Mortalitätsrisikos von Kiefernbeständen in Nordostdeutschland. Nachfolgend werden die Kernergebnisse der jeweiligen Beiträge dieser Dissertation zusammengefasst und interpretiert. So werden diese in den richtigen Forschungskontext eingeordnet, um daraus Aussagen über die Anwendung von satellitenbasierten Wetterindexversicherungen in der Land- und Forstwirtschaft abzuleiten, bevor abschließend ein Ausblick auf weitere notwendige Forschungsfelder gegeben wird. 
Die Anfälligkeit von Kleinbauern für die Auswirkungen von ungünstigen Wetterereignissen ist eines der Haupthindernisse für eine ausreichende Kreditversorgung in weniger entwickelten Ländern wie Madagaskar (Barnett et al., 2008; Giné und Yang, 2009; Farrin und Miranda, 2015; Carter et al., 2011). Dabei ist der Zugang zu Krediten für Kleinbauern neben anderen Faktoren entscheidend für die Produktion und somit die Produktivität der angebauten Kulturen. Allerdings führen wetterbedingte Ertragseinbußen zu Einkommensschwankungen in deren Folge auch die Kreditrückzahlungsfähigkeit der Kleinbauern sinkt (Binswanger und Rosenzweig, 1986). In diesem Zusammenhang untersucht der erste Beitrag dieser Dissertation die Erklärungskraft der Satellitenindizes VCI, TCI und VHI für das Kreditausfallrisiko von landwirtschaftlichen Mikrokrediten von vier einzelnen Filialen der AccèsBanque Madagaskar (ABM, 2016), die landwirtschaftliche Mikrokredite an Reisbauern vergibt. Da das vollständige Ausbleiben der Kredittilgung bei dem untersuchten Mikrofinanzinstitut nahezu nicht auftritt, wurde das Risiko einer verzögerten Kreditrückzahlung als Proxy für das Kreditausfallrisiko bestimmt. Unter Verwendung von Sequential Logit Modellen und Quantilsregressionen wurde neben Kreditmerkmalen und soziodemografischen Eigenschaften der Kreditnehmer der Einfluss des VCI, TCI und VHI auf das Kreditrückzahlungsverhalten von kleinbäuerlichen Reisbauern geschätzt. Die Ergebnisse zeigen, dass die höchste Korrelation zwischen den Indizes VCI, TCI und VHI und den betrachteten Kreditrisikoindikatoren in den für die Ertragsbildung entscheidenden vegetativen und reproduktiven Wachstumsphasen des Reises besteht (Vergara, 1991). Diese Tatsache bestätigt, dass die Bauern die Einnahmen aus dem Verkauf des geernteten Reises zur Rückzahlung ihrer Kredite nutzen und dadurch die wetterbedingten Ertragsschwankungen die Kreditrückzahlungsfähigkeit der Kleinbauern beeinflussen. Die weiteren Ergebnisse bestätigen, dass das Kreditausfallrisiko der Mikrokredite zu einem großen Teil durch die Satellitenindizes VCI, TCI und VHI erklärt werden kann. Darüber hinaus zeigen die Ergebnisse der Quantilsregressionen, dass mit zunehmendem Kreditausfallrisiko die Erklärungskraft der Satellitenindizes steigt.

In diesem Zusammenhang könnte die Versicherung des Agrarkreditportfolios von Mikrofinanzinstituten dazu beitragen, fehlende Ratenzahlungen in Jahren mit extremen Witterungsbedingungen $\mathrm{zu}$ kompensieren und somit die landwirtschaftliche Kreditvergabe zu verbessern (Collier, 2019). Dabei kann die satellitenbasierte Wetterindexversicherung helfen, die Resilienz von Kleinbauern gegenüber Wetterextremen zu stärken, um somit die 
Armut zu bekämpfen und die wirtschaftliche Entwicklung zu beschleunigen (Castillo et al., 2016). Eine solche Indexversicherung könnte folgendermaßen funktionieren: Das Mikrofinanzinstitut schließt Pauschalversicherungen für alle kleinbäuerlichen Kreditnehmer ab. Wenn der zugrunde gelegte Satellitenindex unter ein bestimmtes Strike-Level (Schwellenwert) fällt, zahlt die Versicherung dem Kreditgeber eine Entschädigung. Der Kreditnehmer würde dann von seiner Kreditzahlungsverpflichtung in Höhe der Entschädigungszahlung befreit werden (Shee und Turvey, 2012). Die Kosten für die Versicherung würden von dem Mikrofinanzinstitut getragen werden, wodurch sich zunächst die Zinssätze auf die vergebenen Kredite erhöhen könnten (Shee et al., 2019). Angesichts sinkender Kreditausfallraten kann das Mikrofinanzinstitut die Zinssätze schließlich senken, was den Zugang der Bauern zu Krediten verbessern könnte (Carter et al., 2011; Collier und Skees, 2012; Platteau et al., 2017). Außerdem könnte auch das Mikrofinanzinstitut von niedrigeren Zinssätzen für ihr geliehenes Fremdkapital profitieren und könnte infolgedessen das Kreditangebot und -volumen erhöhen (Collier, 2019).

Enenkel et al. (2019) unterstützen die Ergebnisse des ersten Beitrages dieser Dissertation und stellen fest, dass fernerkundete Satellitenindizes ein hohes Potential haben, schwere Dürren für große Teile Afrikas zu erkennen. Ein wesentlicher Vorteil von Satellitenindizes ist, dass ihre Genauigkeit nicht von der Dichte und Verteilung von Wetterstationen abhängt, die in weniger entwickelten Ländern oft gering ist (Meroni et al., 2013). Somit könnte eine Versicherung auf Basis von Satellitenindizes besonders wertvoll für ein Mikrofinanzinstitut zur Absicherung des Kreditausfallrisikos von Kleinbauern in Entwicklungsländern sein. Im Hinblick auf die Gestaltung der Versicherung erscheint es sinnvoll, ein Strike-Level für die Versicherung im unteren Bereich der zugrundeliegenden Satellitenindizes zu wählen. Somit sollte die Versicherung nur Entschädigungen auszahlen, wenn extreme Wetterbedingungen vorherrschen (Conradt et al., 2015; Weber et al., 2015). Dieses Versicherungsdesign hält die Verwaltungskosten niedrig und ermutigt Kleinbauern dennoch, eigene Risikomanagementstrategien zu verfolgen. $\mathrm{Zu}$ einer weiteren Generalisierbarkeit der Ergebnisse würden längere Zeitreihen von Kreditdatensätzen und die Untersuchung anderer Teile Afrikas in zukünftigen Studien beitragen. Um die Genauigkeit der Erklärungskraft von Satellitenindizes weiter zu erhöhen, könnte sich die zukünftige Forschung auf die Verwendung von Satellitendaten mit einer höheren Auflösung konzentrieren. 
Vor dem Hintergrund eines hohen Potentials von räumlich höher auflösenden Satellitendaten untersucht der zweite Beitrag dieser Dissertation den Einfluss von hochauflösenden Satellitenaufnahmen auf die Hedgingeffektivität von satellitenbasierten Wetterindexversicherungen zur Absicherung des Winterweizenertragsrisikos. Durch die hohe räumliche Auflösung der verwendeten MODIS (250 x $250 \mathrm{~m})$ und Landsat 5/8 (30 x $30 \mathrm{~m})$ Satellitendaten ist es möglich, die Pflanzenvegetation ohne störende Landschaftselemente abzubilden und die Satellitenindizes schlagbezogen zu berechnen. Für drei landwirtschaftliche Betriebe in Nordostdeutschland, das aufgrund sandiger Böden und geringer Niederschläge einem hohen Wetterrisiko ausgesetzt ist, untersucht der zweite Beitrag, ob eine zunehmende räumliche Auflösung der Satellitenindizes das Basisrisiko der räumlichen Auflösung von satellitenbasierten Wetterindexversicherungen reduziert. Weiterhin wird untersucht, ob es einen Aggregationseffekt zwischen dem Design der satellitenbasierten Indexversicherungen auf Schlag- und Betriebsebene gibt, der in früheren Studien zu wetterstationsbasierten Wetterindexversicherungskontrakten beobachtet wurde (Finger, 2012; Heimfarth et al., 2012; Woodard und Garcia, 2008). Als Grundlage für die hypothetisch gestalteten Wetterindexversicherungskontrakte wurde der NDVI als Index verwendet. Die Ergebnisse zeigen, dass mit zunehmender räumlicher Auflösung der Satellitenaufnahmen die Hedgingeffektivität von satellitenbasierten Wetterindexversicherungskontrakten zunimmt, was zu einem geringeren Basisrisiko der räumlichen Auflösung führt (Möllmann et al., 2019). Darüber hinaus wurde auch ein Aggregationseffekt bei satellitenbasierten Wetterindexversicherungskontrakten beobachtet. Versicherungskontrakte auf Betriebsebene zeigen eine höhere Hedgingeffektivität als Versicherungskontrakte auf Schlagebene. Dennoch kann die Gestaltung von satellitenbasierten Wetterindexversicherungskontrakten auf Betriebsebene zu einer Unterschätzung des Risikos führen, da die Datenaggregation die Schwankung der Winterweizenerträge auf Betriebsebene im Vergleich zur Schlagebene reduziert. In diesem Zusammenhang haben Indexversicherungskontrakte, die auf räumlich höher aufgelösten schlagbasierten Landsat-Aufnahmen basieren, mit $29 \%$ eine höhere durchschnittliche Hedgingeffektivität als Indexversicherungskontrakte auf Betriebsebene, die auf MODISAufnahmen basieren (25\%). Daraus lässt sich schließen, dass eine höhere räumliche Auflösung der Satellitenbilder das Basisrisiko der räumlichen Auflösung einer satellitenbasierten Wetterindexversicherung reduziert, während der erhebliche Mehraufwand der Indexbestimmung auf Schlagebene keine Vorteile hinsichtlich der Hedgingeffektivität bringt. 
Im Hinblick auf die Entwicklung und Gestaltung von satellitenbasierten Wetterindexversicherungskontrakten sollten satellitenbasierte Wetterindexversicherungskontrakte auf der Basis von hochauflösenden Satellitenbildern gestaltet werden, um das Basisrisiko der räumlichen Auflösung zu reduzieren. Andererseits ist je nach Stärke des Aggregationseffektes die Frage zu stellen, ob die Indexversicherungskontrakte auf Schlag- oder auf Betriebsebene angeboten werden sollten. Popp et al. (2005) postulieren, dass im Falle eines starken Aggregationseffektes die Erträge auf Schlag- und nicht auf Betriebsebene versichert werden sollten. Ein satellitenbasierter Indexversicherungskontrakt auf Basis von schlagbezogenen Ertragsdaten könnte größere Betriebe, die aufgrund der geringeren Variabilität der Erträge auf Betriebsebene weniger risikoexponiert sind, zur Teilnahme an Versicherungsprogrammen motivieren. Somit würde die Problematik der adversen Selektion durch ein solches Design reduziert werden. Finger (2012) zeigt außerdem, dass bei einem steigenden Aggregationsniveau neben der Ertragsvariabilität auch die fairen Versicherungsprämien für Indexversicherungen sinken würden. Knight et al. (2010) schlagen in diesem Zusammenhang eine Senkung der Versicherungsprämien vor, wenn das versicherte Aggregationsniveau steigt (z. B. von der Schlag- zur Betriebsebene) und wenn die Größe der versicherten Einheit zunimmt.

Die Ergebnisse dieses zweiten Beitrages legen nahe, dass die Verwendung von hochauflösenden Satellitenbildern das Basisrisiko der räumlichen Auflösung von satellitenbasierten Wetterindexversicherungen weiter reduziert. Dennoch ist das teilweise Auftreten von Bewölkung in den Landsat Satellitenaufnahmen eine Limitation solch designter Indexversicherungskontrakte. Daher sollten zukünftige Studien, sobald es die Länge der Datenzeitreihe erlaubt, Sentinel 2 Satellitenaufnahmen mit hoher zeitlicher und räumlicher Auflösung verwenden und insbesondere die Anwendbarkeit von aktiven Radarsatelliten wie Sentinel 1 mit einem Synthetic Aperture Radar (SAR) für Versicherungszwecke untersuchen, da radarbasierte Sensoren unabhängig von der Bewölkung Informationen über den Vegetationszustand von Pflanzenbeständen liefern können. Darüber hinaus könnten auch andere Indizes wie der Temperature Condition Index (TCI) im Hinblick auf unterschiedliche räumliche Auflösungen untersucht werden. In diesem Zusammenhang sollten zukünftige Studien auch die Höhe der Versicherungsprämie für satellitenbasierte Indexversicherungen in Abhängigkeit von der Größe des versicherten Betriebes und dem Aggregationsgrad un- 
tersuchen, da Versicherungsparameter, die auf aggregierten Ertragsdaten basieren, zu einer Unterschätzung des Ertragsrisikos führen können.

Da insbesondere bei mehrjährigen Plantagenkulturen die Anpassungsfähigkeit an den voranschreitenden Klimawandel durch den längeren Lebenszyklus und die erhöhten Investitionskosten erschwert ist (Gunathilaka et al., 2018), untersucht der dritte Beitrag dieser Dissertation die Möglichkeit von satellitenbasierten Wetterindexversicherungskontrakten zu Absicherung des Olivenölertrages von mehrjährigen, nicht-bewässerten Olivenbäumen in Andalusien. Obwohl Olivenbäume gut an geringe Niederschläge angepasst sind, wird häufig die Bewässerung in Andalusien eingesetzt, um die Olivenölertragsschwankungen zu reduzieren. Da allerdings das Wasser zunehmend $\mathrm{zu}$ einer knappen Ressource wird (Martínez und Almonacid, 2017), könnten satellitenbasierte Wetterindexversicherungen eine interessante Alternative zur Bewässerung darstellen. Daher werden in dem dritten Beitrag satellitenbasierte Wetterindexversicherungskontrakte für alle unbewässerten Olivenfelder in fünf Provinzen Andalusiens entworfen. Zusätzlich zu den verwendeten Satellitenindizes VCI, TCI und VHI werden Niederschlags- und Temperaturindizes als Benchmarkindizes betrachtet. Die Satellitenindizes werden auf Grundlage von MODIS Satellitenaufnehmen mit einer räumlichen Auflösung von 250 x 250 m auf Provinzebene berechnet. Darüber hinaus wird ein Copula-Ansatz verwendet, um die Beziehung zwischen dem Olivenölertrag und den Indizes flexibler modellieren zu können und um somit Aussagen über das Basisrisiko des Designs machen zu können.

Die Ergebnisse des dritten Beitrages zeigen, dass die stärkste Beziehung zwischen den Olivenölerträgen und den betrachten Indizes in den Phasen der Blütenstandsentwicklung, der Blüte, des Fruchtwachstums und der Ölakkumulation besteht. Bei Verwendung der Gumbel Copula besitzen die VCI- und VHI-basierten Versicherungskontrakte eine durchschnittliche Hedgingeffektivität von $38 \%$ und übertreffen temperatur- und niederschlagsbasierte Versicherungskontrakte (16 \% bzw. 29 \%). Mit einer durchschnittlichen Hedgingeffektivität von $29 \%$ sind auch TCI-basierte Versicherungskontrakte besser als temperaturbasierte Versicherungskontrakte geeignet. Dennoch gibt es zwischen den jeweiligen Provinzen Unterschiede in der Höhe der Hedgingeffektivitäten. Während VCI-basierte Versicherungskontrakte tendenziell eine starke Hedgingeffektivität für Provinzen in niedrigeren Höhenlagen haben, scheinen TCI- und VHI-basierte Versicherungskontrakte eher 
für Provinzen in bergigen Höhenlagen geeignet zu sein. Weiterhin kann anhand der Ergebnisse darauf geschlossen werden, dass satellitenbasierte Versicherungskontrakte ein geringeres Basisrisiko haben als die meteorologisch-basierten Versicherungskontrakte.

Der dritte Beitrag macht einen ersten Vorschlag zur Absicherung des hohen Ertragsrisikos von mehrjährigen Plantagenkulturen wie z.B. Olivenbäumen durch eine satellitenbasierte Wetterindexversicherung. Die Studie zeigt, dass satellitenbasierte Wetterindexversicherungskontrakte die Ertragsschwankungen von unbewässerten Olivenhainen durch Entschädigungszahlungen absichern können. Daher sind der Fokus und die Ergebnisse des dritten Beitrages nicht nur für Olivenbauern interessant, sondern auch für politische Entscheidungsträger. So werden in Zukunft Alternativen zur Bewässerung von Olivenhainen in Andalusien notwendig sein, da die Verfügbarkeit von Wasser aufgrund eines verstärkten Anbaus von bewässerten Olivenbäumen immer problematischer wird. Daher konzentriert sich die politische Agenda für die Budgetzuweisungen in Spanien auf die Stärkung einer effizienten und nachhaltigen Produktion, den Erhalt natürlicher Ressourcen und die Begrenzung ihrer Ausbeutung (Martínez und Almonacid, 2017). Quiroga und Iglesias (2009) weisen jedoch darauf hin, dass die Förderung von Versicherungen im Rahmen der Gemeinsamen Agrarpolitik (GAP) bessere Methoden zur Quantifizierung der Beziehung zwischen Klima und Ertragsverhalten erfordert. Versicherungskontrakte, die anhand der Gumbel-Copula designt wurden, besitzen eine höhere Hedgingeffektivität im Vergleich zu Versicherungskontrakten auf Grundlage der Gauß-Copula. Folglich kann durch die Verwendung der Gumbel-Copula, die die Beziehung zwischen Ertrag und Index aufgrund einer stärkeren Abhängigkeitsstruktur im linken Bereich der gemeinsamen Verteilungsfunktion besser abbilden kann, das Basisrisiko des Designs von satellitenbasierten Indexversicherungskontrakten reduziert werden, womit diese Studie auch für potentielle Anbieter von Wetterindexversicherungen eine Relevanz besitzt.

Allerdings hängt die Wirkung von satellitenbasierten Wetterindexversicherungen stark von der Qualität der Satelliten- und Ertragsdaten ab. Für zukünftige Untersuchungen sollten daher weniger stark aggregierte Daten als Grundlage für die Olivenölerträge dienen. Die Hedgingeffektivität der satellitenbasierten Wetterindexversicherungskontrakte wird sich vermutlich noch verbessern, da durch die Verwendung von Durchschnittswerten auf Provinzebene ein natürlicher Risikoausgleich innerhalb der Provinzen besteht. Weiterhin muss 
erwähnt werden, dass die genaue Lage der Olivenhaine nicht bekannt ist. Die Identifizierung der Olivenfelder hat eine Genauigkeit von etwa $85 \%$ (Land Monitoring Service, 2012). Die Verwendung von Satellitendaten mit einer höheren räumlichen Auflösung könnte jedoch die Genauigkeit der Identifizierung der Olivenfelder weiter verbessern. Darüber hinaus könnten in weiteren Studien alternative Satellitenindizes wie der Normalized Difference Red Edge (NDRE) untersucht werden. Besonders bei immergrünen Bäumen, die einen nahezu stabilen Chlorophyll-Gehalt während des Jahres beibehalten, schätzt der NDRE wahrscheinlich einen größeren Bereich des Chlorophyll-Gehaltes (Lupidi und Masini, 2015).

Abseits der Landwirtschaft konnte gerade in den letzten Jahren eine starke Auswirkung längerer Hitzeperioden auf den Zustand von Wäldern beobachtet werden. Obwohl die Vitalität von Bäumen stark von extremen Wetterbedingungen beeinflusst wird (Beck und Heinzig, 2018), gibt es in vielen Ländern keine Versicherungen, die zu angemessenen Versicherungsprämien das jährliche Wachstumsrisiko von stehendem Holz absichern. Aufgrund des Fehlens von zuverlässigen Methoden zur Quantifizierung von Waldwachstumsrisiken ist es problematisch, das Ausmaß möglicher Schäden im Forstsektor abzuschätzen (Holecy und Hanewinkel, 2006; Pinheiro und Ribeiro, 2013). Dadurch können häufig keine kostengünstigen Versicherungslösungen angeboten werden. In diesem $\mathrm{Zu}$ sammenhang könnten Indexversicherungen interessant sein, da sich mögliche Schäden direkt aus dem Indexwert ableiten lassen. Daher verursachen Indexversicherungen im Vergleich zu traditionellen schadensbasierten Versicherungen geringere Transaktionskosten (Collier et al., 2009). Vor diesem Hintergrund untersucht der vierte Beitrag die Möglichkeit von satellitenbasierten Indexversicherungen zur Absicherung des jährlichen Vitalitätsbzw. Mortalitätsrisikos von zwölf verschiedenen Kiefernbeständen in Nordostdeutschland. Neben den Satellitenindizes VCI, TCI und VHI auf Grundlage von MODIS Satellitendaten werden Benchmarkindizes in Form der Niederschlagsmenge, der Temperaturhöhe und eines kombinierten Niederschlags-Temperatur-Indizes (PTI) berechnet. Über einen Baumringindex (TRI) wird schließlich der jährliche Vitalitätszustand der Wälder bestimmt.

Zwischen dem TRI und den jeweilig untersuchten Indizes besteht ein hoher Zusammenhang. So konnte für den TCI und die Temperatur der höchste Korrelationskoeffizient mit dem TRI im Sommer beobachtet werden. Dagegen besteht zwischen dem VCI bzw. dem 
Niederschlag und dem TRI in der gesamten Vegetationsperiode ein hoher Zusammenhang. Weiterhin wird aus den designten Indexversicherungskontrakten deutlich, dass sowohl die wetterstations- als auch die satellitenbasierten Indexversicherungskontrakte das Mortalitätsrisiko von Kiefernbeständen absichern können. Im Durchschnitt aller untersuchten Kiefernbestände weisen die niederschlags-, temperatur- und PTI-basierten Versicherungskontrakte eine durchschnittliche Hedgingeffektivität von $40 \%, 41 \%$ und $47 \%$ auf. Die satellitenbasierten Versicherungskontrakte besitzen für den VCI (45\%), TCI (36 \%) und VHI (48\%) ähnliche durchschnittliche Hedgingeffektivitäten.

Die Ergebnisse des vierten Beitrages deuten darauf hin, dass Indexversicherungskontrakte eine Möglichkeit zur Risikoabschätzung und zur Absicherung des Mortalitätsrisikos von Kiefernbeständen bieten können. Daher könnten diese Ergebnisse sowohl für Waldbesitzer als auch für potentielle Anbieter von Wetterindexversicherungen interessant sein. Insbesondere Satellitenindizes bieten den Vorteil, dass sie nicht an Wetterstationen gemessen werden müssen und teilweise kostenlos für jeden Ort der Welt verfügbar sind, wodurch die Satellitenindizes auch für große Waldgebiete berechnet werden können. Durch Indexversicherungen könnten die hohen Versicherungsprämien in der Forstwirtschaft möglicherweise reduziert werden. In Folge niedriger Versicherungsprämien könnte auch die Nachfrage seitens der Waldbesitzer nach Versicherungskontrakten steigen. Aber auch für politische Entscheidungsträger sind die Ergebnisse interessant. Denn Versicherungslösungen sind den öffentlichen Entschädigungszahlungen bei hohen Schadensereignissen, die in vielen Ländern üblich sind, weitgehend überlegen, da sie den versicherten Waldbesitzern stärkere Anreize geben, Risiken entgegenzuwirken (Chen et al., 2014). So verringern aber gerade diese öffentlichen Entschädigungen die Anreize der Waldbesitzer, ihre Wälder zu versichern (Holecy und Hanewinkel, 2006; Brunette und Couture, 2008; Sauter et al., 2016). Im Gegensatz zu den unspezifischen, öffentlichen Entschädigungsauszahlungen könnten Versicherungslösungen flächendeckender angeboten werden (Chen et al., 2014). Wie bereits einige Studien zeigen konnten, würden dann die Versicherungsprämien mit zunehmender Größe des Versicherungsmarktes und des Versicherungsgebietes sinken (Holecy und Hanewinkel, 2006; Brunette et al., 2015).

Nichtsdestotrotz ist diese Studie als erstes Anwendungsbeispiel für die Übertragung von satellitenbasierten Wetterindexversicherungen vom landwirtschaftlichen Bereich auf den 
forstwirtschaftlichen Sektor zu sehen. In der durchgeführten Wirkungsanalyse von Indexversicherungskontrakten zur Absicherung des Mortalitätsrisikos von Kiefernbeständen wird der Jahrringindex als Proxy für die jährliche Vitalität der Bäume verwendet. Dabei kann jedoch kritisch hinterfragt werden, ob der Jahrringindex als alleiniges Maß die Jahresvitalität von Bäumen widerspiegeln kann. Vor dem Hintergrund einer schwierigen Bewertung des realen Wertes von Waldbeständen aufgrund unterschiedlicher Altersstrukturen und teilweise unterschiedlicher Baumarten (Pinheiro und Ribeiro, 2013) könnten sich zukünftige Studien zu satellitenbasierten Indexversicherungen im Forstbereich mit anderen Proxy-Variablen als dem Jahrringindex für die Beurteilung des Vitalitätszustandes der Bäume beschäftigen. Weiterhin sollten zukünftige Studien neben der Kiefer noch weitere Baumarten untersuchen. Weitere Ansatzpunkte für nachfolgende Studien sind die Verwendung anderer Indizes wie satellitenbasierter Niederschlagsindizes oder die Berechnung der Satellitenindizes auf Grundlage höher auflösender Satellitendaten.

Zusammenfassend lässt sich festhalten, dass die vier Beiträge dieser Dissertation das Potential von satellitenbasierten Wetterindexversicherungen sowohl für die Land- als auch Forstwirtschaft aufzeigen. Dabei eignen sich satellitenbasierte Indexversicherungskontrakte, trockenheitsbedingte Schäden in einjährigen Kulturen wie Reis und Weizen, in langjährigen Olivenbäumen sowie in langjährigen Kiefernbeständen abzusichern. Die Satellitenindizes im Rahmen von Wetterindexversicherungen können eine Möglichkeit bieten, das Problem des geografischen Basisrisikos und des Basisrisikos der Produktion im Vergleich zu meteorologischen Wetterstationsindizes zu reduzieren. Darüber hinaus kann das Basisrisiko der räumlichen Auflösung von satellitenbasierten Versicherungskontrakten durch höher auflösendere Satellitenaufnahmen reduziert werden. Durch ein Design der Versicherungskontrakte auf Grundlage des Copula-Ansatzes oder von Quantilsregressionen kann weiterhin das Basisrisiko des Designs abgeschwächt werden. Daher sind die Ergebnisse dieser Dissertation für einen breiten Adressatenkreis aus der praktischen Land- und Forstwirtschaft, aus dem Bereich der Versicherungsgesellschaften aber auch aus dem Kreis der politischen Entschädigungsträger interessant. Dabei könnte gerade eine politische Unterstützung in Form von Subventionen für eine schnellere und flächendeckendere Implementierung solcher satellitenbasierten Indexversicherungen sorgen. Satellitenbasierte Indexversicherungen könnten als Risikomanagementinstrumente zur Anpassung an den voranschreitenden Klimawandel genutzt werden. Diesbezüglich wird bereits im Rahmen einer 
neuen EU-Strategie zur Anpassung an den Klimawandel ein Klimaversicherungsschutz untersucht, diskutiert und wenn möglich auch gefördert (Europäische Kommission, 2021). Zukünftig besteht aber zusätzlicher Forschungsbedarf. Einerseits muss weiterhin die Hedgingeffektivität der satellitenbasierten Indexversicherungskontrakte verbessert werden. Hierbei könnten zukünftige Studien Satellitenaufnahmen der Sentinel 2 und Sentinel 1 Satelliten verwenden, um zum einen die zeitliche und räumliche Auflösung der Satellitenaufnahmen zu erhöhen und um zum anderen unabhängig von der Bewölkung zu sein. Da mit steigender Auflösung der Satellitenaufnahmen allerdings die Probleme von Moral Hazard und der adversen Selektion wieder auftreten können, könnten auch andere Indizes wie ein satellitenbasierter Bodenfeuchteindex im Rahmen von Wetterindexversicherungen interessant sein (Vroege et al., 2021). In diesem Zusammenhang bietet zum Beispiel die Firma VanderSat (2021) schwerpunktmäßig satellitenbasierte Bodenfeuchtigkeitsdaten an. Darüber hinaus könnten zukünftige Studien das zeitliche Basisrisiko (Dalhaus und Finger, 2016; Dalhaus et al., 2018) von satellitenbasierten Wetterindexversicherungen näher untersuchen. Auf der Basis von Satellitendaten könnten kritische Indexberechnungszeiträume und somit die jährlich variierenden kritischen Phasen des Pflanzenertrages bestimmt werden, um die jährlichen Schwankungen beim Beginn und dem Ende der Vegetationsperiode im Rahmen einer satellitenbasierten Indexversicherung berücksichtigen zu können. Da andererseits neben den in dieser Dissertation durchgeführten Wirkungsanalysen auch andere Aspekte für die praktische Umsetzung solcher Indexversicherungen wichtig sind, sollten nachfolgende Studien auch ökonomische Fragen wie die Zahlungsbereitschaft, die kulturspezifische Versicherungssumme, die Versicherungsprämie aber auch die Höhe des StrikeLevels für satellitenbasierte Indexversicherungskontrakte untersuchen. 


\section{Literatur}

ABM (2016): Annual Report. AccèsBanque Madagascar. Online verfügbar unter https://www.accesbanque.mg/wp-content/uploads/2018/01/171123-RA-AccèsBanque-2016_Correct.pdf, zuletzt geprüft am 06.05.2021.

Barnett, B. J.; Barrett, C. B.; Skees, J. R. (2008): Poverty Traps and Index-Based Risk Transfer Products. In: World Development 36 (10), S. 1766-1785.

Beck, W.; Heinzig, P. (2018): A new tool to discovering realistic climate-growth relationships. In: FREIJ 2 (2). DOI: 10.15406/freij.2017.02.00025.

Binswanger, H. P.; Rosenzweig, M. R. (1986): Behavioural and material determinants of production relations in agriculture. In: The Journal of Development Studies 22 (3), S. 503-539. DOI: 10.1080/00220388608421994.

Brunette, M.; Holecy, J.; Sedliak, M.; Tucek, J.; Hanewinkel, M. (2015): An actuarial model of forest insurance against multiple natural hazards in fir (Abies Alba Mill.) stands in Slovakia. In: Forest Policy and Economics 55 (2), S. 46-57. DOI: 10.1016/j.forpol.2015.03.001.

Brunette, M.; Couture, S. (2008): Public compensation for windstorm damage reduces incentives for risk management investments. In: Forest Policy and Economics 10 (78), S. 491-499. DOI: 10.1016/j.forpol.2008.05.001.

Carter, M. R.; Cheng, L.; Sarris, A. (Hg.) (2011): The impact of interlinked index insurance and credit contracts on financial market deepening and small farm productivity. Annual Meeting of the American Applied Economics Association, Pittsburgh PA, July. Pittsburgh PA.

Castillo, M. J.; Boucher, S.; Carter, M. (2016): Index Insurance: Using Public Data to Benefit Small-Scale-Agriculture. In: International Food and Agribusiness Management Review Special Issue (19 Issue A).

Chen, X.; Goodwin, B. K.; Prestemon, J. P. (2014): Is Timber Insurable? A Study of Wildfire Risks in the U.S. Forest Sector Using Spatio-temporal Models. In: American Journal of Agricultural Economics 96 (1), S. 213-231. DOI: 10.1093/ajae/aat087.

Collier, B. (2019): Strengthening Local Credit Markets Through Lender-Level Index Insurance. In: Journal of Risk and Insurance, S. 2757. DOI: 10.1111/jori.12277. 
Collier, B.; Skees, J. (2012): Increasing the resilience of financial intermediaries through portfolio-level insurance against natural disasters. In: Natural hazards 64 (1), S. 55-72. DOI: 10.1007/s11069-012-0227-0.

Collier, B.; Skees, J.; Barnett, B. (2009): Weather Index Insurance and Climate Change: Opportunities and Challenges in Lower Income Countries. In: Geneva Pap Risk Insur Issues Pract 34 (3), S. 401-424. DOI: 10.1057/gpp.2009.11.

Conradt, S.; Finger, R.; Bokusheva, R. (2015): Tailored to the extremes: Quantile regression for index-based insurance contract design. In: Agricultural Economics 46 (4), S. 537-547. DOI: 10.1111/agec.12180.

Dalhaus, T.; Finger, R. (2016): Can Gridded Precipitation Data and Phenological Observations Reduce Basis Risk of Weather Index-Based Insurance? In: Wea. Climate Soc. 8 (4), S. 409-419. DOI: 10.1175/WCAS-D-16-0020.1.

Dalhaus, T.; Mußhoff, O.; Finger, R. (2018): Phenology Information Contributes to Reduce Temporal Basis Risk in Agricultural Weather Index Insurance. In: Scientific reports 8 (1), S. 46. DOI: 10.1038/s41598-017-18656-5.

Enenkel, M.; Osgood, D.; Anderson, M.; Powell, B.; McCarty, J.; Neigh, C. et al. (2019): Exploiting the Convergence of Evidence in Satellite Data for Advanced Weather Index Insurance Design. In: Wea. Climate Soc. 11 (1), S. 65-93. DOI: 10.1175/WCAS-D-17-0111.1.

Europäische Kommission (2021): Ein klimaresilientes Europa aufbauen - die neue EUStrategie für die Anpassung an den Klimawandel. Mitteilung der Kommission an das Europäische Parlament, den Rat, den europäischen Wirtschafts- und Sozialausschuss und den Ausschuss der Regionen. Online verfügbar unter https://eurlex.europa.eu/legal-content/EN/TXT/PDF/?uri=CELEX:52021DC0082\&from=EN, zuletzt geprüft am 10.05.2021.

Farrin, K.; Miranda, M. J. (2015): A heterogeneous agent model of credit-linked index insurance and farm technology adoption. In: Journal of Development Economics 116, S. 199-211. DOI: 10.1016/j.jdeveco.2015.05.001.

Finger, R. (2012): Biases in Farm-Level Yield Risk Analysis due to Data Aggregation. DOI: 10.3929/ethz-b-000061653. 
Giné, X.; Yang, D. (2009): Insurance, credit, and technology adoption: Field experimental evidence from Malawi. In: Journal of Development Economics 89 (1), S. 1-11. DOI: 10.1016/j.jdeveco.2008.09.007.

Gunathilaka, R. P. D.; Smart, J. C. R.; Fleming, C. M. (2018): Adaptation to climate change in perennial cropping systems: Options, barriers and policy implications. In: Environmental Science \& Policy 82, S. 108-116. DOI: 10.1016/ j.envsci. 2018.01.011.

Heimfarth, L. E.; Finger, R.; Mußhoff, O. (2012): Hedging weather risk on aggregated and individual farm-level. In: Agricultural Finance Review 72 (3), S. 471-487. DOI: $10.1108 / 00021461211277295$.

Holecy, J.; Hanewinkel, M. (2006): A forest management risk insurance model and its application to coniferous stands in southwest Germany. In: Forest Policy and Economics 8 (2), S. 161-174. DOI: 10.1016/j.forpol.2004.05.009.

Land Monitoring Service (2012): Copernicus-CORINE land cover. CLC 2012. Online verfügbar unter https://land.copernicus.eu/pan-european/corine-land-cover, zuletzt geprüft am 28.01.2019.

Lupidi, A.; Masini, A. (2015): Remote Sensing data and analysis. WP 2 Forest information collection and analysis. In: SLOPE (Integrated proceSsing and controL systems fOr sustainable forest Production in mountain arEas).

Martínez, J. D. S.; Almonacid, A. G. (2017): Productivism and Post-Productivism in the Olive Groves of Southern Spain. In: Quaestiones Geographicae 36 (2), S. 57-69. DOI: 10.1515/quageo-2017-0015.

Meroni, M. J.; Kayitakire, F.; Brown, M. (2013): Remote sensing of vegetation: potential applications for index insurance. In: R. Gommes und F. Kayitakire (Hg.): The challenges of index-based insurance for food security in developing countries. Luxembourg, S. 238-245.

Möllmann, J.; Buchholz, M.; Mußhoff, O. (2019): Comparing the Hedging Effectiveness of Weather Derivatives Based on Remotely Sensed Vegetation Health Indices and Meteorological Indices. In: Weather, Climate, and Society 11 (1), S. 33-48. DOI: 10.1175/WCAS-D-17-0127.1.

Pinheiro, A. C.; Ribeiro, N. (2013): Forest property insurance: an application to Portuguese woodlands. In: IJSSOC 5 (3), S. 284. DOI: 10.1504 /IJSSOC.2013.054716. 
Platteau, J.-P.; Bock, O.; Gelade, W. (2017): The Demand for Microinsurance: A Literature Review. In: World Development 94, S. 139-156. DOI: 10.1016/j.worlddev.2017.01.010.

Popp, M.; Rudstrom, M.; Manning, P. (2005): Spatial Yield Risk Across Region, Crop and Aggregation Method. In: Canadian J Agric Econ 53 (2-3), S. 103-115. DOI: 10.1111/j.1744-7976.2005.00408.x.

Quiroga, S.; Iglesias, A. (2009): A comparison of the climate risks of cereal, citrus, grapevine and olive production in Spain. In: Agricultural Systems 101 (1-2), S. 91-100. DOI: 10.1016/j.agsy.2009.03.006.

Sauter, P. A.; Möllmann, T. B.; Anastassiadis, F.; Mußhoff, O.; Möhring, B. (2016): To insure or not to insure? Analysis of foresters' willingness-to-pay for fire and storm insurance. In: Forest Policy and Economics 73 (2), S. 78-89. DOI: 10.1016/j.forpol.2016.08.005.

Shee, A.; Turvey, C. G. (2012): Collateral-free lending with risk-contingent credit for agricultural development: indemnifying loans against pulse crop price risk in India. In: Agricultural Economics $43 \quad$ (5), S. 561-574. DOI: 10.1111/j.15740862.2012.00603.x.

Shee, A.; Turvey, C. G.; You, L. (2019): Design and rating of risk-contingent credit for balancing business and financial risks for Kenyan farmers. In: Applied Economics 75 (4), S. 1-19. DOI: 10.1080/00036846.2019.1613502.

VanderSat (2021): Unmatched water data. Satellite observed. Detailed soil moisture data. Online verfügbar unter https://vandersat.com/data/soil-moisture/, zuletzt geprüft am 10.05.2021.

Vergara, B. S. (1991): Rice plant growth and development. In: S. L. Bor (Hg.): Rice. 2. Aufl.: Springer, S. 13-22.

Vroege, W.; Bucheli, J.; Dalhaus, T.; Hirschi, M.; Finger, R. (2021): Insuring crops from space: the potential of satellite-retrieved soil moisture to reduce farmers' drought risk exposure. In: European Review of Agricultural Economics 48 (2), S. 266-314. DOI: $10.1093 /$ erae/jbab010.

Weber, R.; Fecke, W.; Moeller, I.; Mußhoff, O. (2015): Meso-level weather index insurance. In: Agricultural Finance Review 75 (1), S. 31-46. DOI: 10.1108 /AFR-122014-0045. 
Woodard, J. D.; Garcia, P. (2008): Weather Derivatives, Spatial Aggregation, and Systemic Risk: Implications for Reinsurance Hedging. In: Journal of Agricultural and Resource Economics 33 (1), S. 34-51. 


\section{Publikationsliste}

\section{Beiträge in referierten wissenschaftlichen Zeitschriften:}

Möllmann, J., Buchholz, M., Kölle, W. \& Mußhoff, O. (2020). Do remotely-sensed vegetation health indices explain credit risk in agricultural microfinance? World Development, Volume 127, March 2020, 104771, https://doi.org/10.1016/j.world dev.2019.104771.

Kölle, W., Martínez Salgueiro A., Buchholz, M. \& Mußhoff, O. (2020). Can satellite-based weather index insurance improve the hedging of yield risk of perennial nonirrigated olive trees in Spain? Australian Journal of Agricultural and Resource Economics, Volume 65, Issue 1, January 2021, Pages 66-93, http://dx. doi.org/ $10.1561 / 112.00000533$.

Kölle, W., Buchholz, M. \& Mußhoff, O. (2021). Can satellite-based weather index insurance hedge the mortality risk of pine stands? Journal of Forest Economics, Vol. 36: No. 4, pp 315-350; http://dx.doi.org/10.1561/112.00000533.

Kölle, W., Buchholz, M. \& Mußhoff, O. (2021). Do high-resolution satellite indices at field level reduce basis risk of satellite-based weather index insurance? Agricultural Finance Review, https://doi.org/10.1108/AFR-12-2020-0177. 


\section{Erklärung über den geleisteten Eigenanteil an der Arbeit}

Hiermit erkläre ich den geleisteten Anteil an den in die Dissertationsschrift aufgenommenen Beiträgen.

Im ersten Beitrag mit dem Titel „Do remotely-sensed vegetation health indices explain credit risk in agricultural microfinance?”, der in Zusammenarbeit mit Herrn Dr. Johannes Möllmann, Herrn Dr. Matthias Buchholz und Herrn Prof. Dr. Oliver Mußhoff verfasst wurde, sind folgende Bereiche von mir übernommen worden: Durchführung der Berechnungen sowie die Interpretation der Ergebnisse in Zusammenarbeit mit Dr. Johannes Möllmann mit Beratung durch Herrn Dr. Matthias Buchholz und Herrn Prof. Dr. Oliver Mußhoff und das Überarbeiten des Beitrages in Zusammenarbeit mit Dr. Matthias Buchholz mit Beratung durch Herrn Prof. Dr. Oliver Mußhoff.

Im zweiten Beitrag mit dem Titel „Do high-resolution satellite indices at field level reduce basis risk of satellite-based weather index insurance?", der in Zusammenarbeit mit Herrn Dr. Matthias Buchholz und Herrn Prof. Dr. Oliver Mußhoff entstanden ist, sind folgende Bereiche von mir übernommen worden: Idee und Konzeption des Beitrages in Zusammenarbeit mit Herrn Dr. Matthias Buchholz, Durchführung der Berechnungen, Analyse und Interpretation der Ergebnisse, Verfassen und Überarbeiten des Beitrages mit Unterstützung durch Herrn Dr. Matthias Buchholz und Beratung durch Herrn Prof. Dr. Oliver Mußhoff.

Im dritten Beitrag mit dem Titel „Can satellite-based weather index insurance improve the hedging of yield risk of perennial non-irrigated olive trees in Spain?", der in Zusammenarbeit mit Frau Dr. Andrea Martínez Salgueiro, Herrn Dr. Matthias Buchholz und Herrn Prof. Dr. Oliver Mußhoff entstanden ist, sind folgende Bereiche von mir übernommen worden: Idee und Konzeption des Beitrages in Zusammenarbeit mit Frau Dr. Andrea Martínez Salgueiro, Durchführung der Berechnungen, Interpretation der Ergebnisse, Verfassen und Überarbeiten des Beitrages in Zusammenarbeit mit Frau Dr. Andrea Martínez Salgueiro mit Beratung durch Herrn Dr. Matthias Buchholz und Herrn Prof. Dr. Oliver Mußhoff. 
Im vierten Beitrag mit dem Titel „Can satellite-based weather index insurance hedge the mortality risk of pine stands?", der in Zusammenarbeit mit Herrn Dr. Matthias Buchholz und Herrn Prof. Dr. Oliver Mußhoff entstanden ist, sind folgende Bereiche von mir übernommen worden: Idee und Konzeption des Beitrages in Zusammenarbeit mit Herrn Dr. Matthias Buchholz, Durchführung der Berechnungen, Analyse und Interpretation der Ergebnisse, Verfassen und Überarbeiten des Beitrages mit Unterstützung durch Herrn Dr. Matthias Buchholz und Beratung durch Herrn Prof. Dr. Oliver Mußhoff. 


\section{Eidesstattliche Erklärungen}

Hiermit erkläre ich eidesstattlich, dass:

1. diese Arbeit weder in gleicher noch in ähnlicher Form bereits anderen Prüfungsbehörden vorgelegen hat.

2. ich mich an keiner anderen Hochschule um einen Doktorgrad beworben habe.

Göttingen, den

(Unterschrift)

Hiermit erkläre ich eidesstattlich, dass diese Dissertation selbständig und ohne unerlaubte Hilfe angefertigt wurde.

Göttingen, den

(Unterschrift) 